\title{
Finite-dimensional piezoelectric transducer modeling for guided wave based structural health monitoring
}

\author{
Ajay Raghavan and Carlos E S Cesnik ${ }^{1}$ \\ Department of Aerospace Engineering, The University of Michigan, 1320 Beal Avenue, \\ Ann Arbor, MI 48109, USA \\ E-mail: cesnik@umich.edu
}

Received 19 November 2004, in final form 13 September 2005

Published 9 November 2005

Online at stacks.iop.org/SMS/14/1448

\begin{abstract}
Among the various schemes being considered for structural health monitoring (SHM), guided wave (GW) testing in particular has shown great promise. While GW testing using hand-held transducers for non-destructive evaluation (NDE) is a well established technology, GW testing for SHM using surface-bonded/embedded piezoelectric wafer transducers (piezos) is relatively in its formative years. Little effort has been made towards a precise characterization of GW excitation using piezos and often the various parameters involved are chosen without mathematical foundation. In this work, a formulation for modeling the transient GW field excited using arbitrary shaped surface-bonded piezos in isotropic plates based on the 3D linear elasticity equations is presented. This is then used for the specific cases of rectangular and ring-shaped actuators, which are most commonly used in GW SHM. Equations for the output voltage response of surface-bonded piezo-sensors in GW fields are derived and optimization of the actuator/sensor dimensions is done based on these. Finally, numerical and experimental results establishing the validity of these models are discussed.
\end{abstract}

(Some figures in this article are in colour only in the electronic version)

\section{Introduction}

The importance of damage prognosis systems in civil, mechanical and aerospace structures has gained growing awareness of late. Such a system would apprise the structural user of the structure's condition, monitor its health by scanning for damage, and provide intelligent estimates regarding the remaining useful life of the structure. The usefulness of such systems would be enormous, and they have tremendous potential to provide huge labor and monetary savings and most importantly will improve confidence and safety levels in operating structures. The gamut of structures that can potentially benefit from the development of such technologies extends from ground vehicles, ships and aerospace structures to bridges, pipelines and offshore platforms. Structural health monitoring (SHM) is a critical component of damage prognosis

\footnotetext{
1 Author to whom any correspondence should be addressed.
}

systems. SHM is the part of the prognosis system that examines the structure for damage and provides information about the same. An SHM system usually consists of an onboard network of sensors for data acquisition and some central processor using a tested algorithm to diagnose structural health.

Among various ideas being explored for SHM, guided wave (GW) testing has shown great potential. The terms 'guided wave' and 'Lamb wave' are often used interchangeably in the literature; however, strictly speaking, Lamb waves refer to just one class of GWs that can be excited in a flat, infinite isotropic plate. The former term is universal and is not confined to any particular class of waves in a particular structural waveguide. GW methods have been used for over two decades in the non-destructive evaluation (NDE) industry. However, the ultrasonic transducers used there are not compact enough to be mounted permanently onboard the structure. In recent years, several researchers have used piezoelectric wafer 
transducers (hereafter referred to as 'piezos') for GW based SHM with encouraging results [1-6].

GW theory is complicated due to the dispersive nature of these waves and the fact that at least two modes can propagate at any frequency. Therefore, a good understanding of GW theory and a grass-roots level characterization of the nature of GWs generated and detected by surface-bonded/embedded piezos is very desirable. The theory of free GW propagation in isotropic, anisotropic and layered materials for various geometries as well as excitation using conventional NDE ultrasonic transducers is well documented [7]. The free GW modes in isotropic plates and shells were first studied by Lamb [8] and Gazis [9] respectively using the theory of elasticity. Earlier works on modeling excitation of GW fields using the theory of elasticity have mostly used 2D models, wherein a 'plane-strain'-like assumption was built into the models. The book by Viktorov [10] on 2D models based on the theory of elasticity for Lamb wave excitation in isotropic plates by NDE transducers was a pioneering work in this direction. The residue theorem was used in that work to invert the 1D Fourier transform integrals. A heuristic model was also proposed for extending the $2 \mathrm{D}$ model to the case of $3 \mathrm{D}$ excitation by NDE transducers. Ditri and Rose [11] used plane strain models along with the normal mode expansion technique to describe GW excitation in composites by NDE transducers. Santosa and Pao [12] solved the generic 3D problem of GW excitation in an isotropic plate by an impulse point body force, also using the normal mode expansion technique. Wilcox [13] presented a 3D elasticity model describing the harmonic GW field by generic surface point sources in isotropic plates; however, the model was not rigorously developed, and some intuitive reasoning was used to extend $2 \mathrm{D}$ model results to $3 \mathrm{D}$. It should be noted that none of these works addressed rigorous modeling of finite-dimensional actuators using 3D elasticity.

Relatively less work has been done for GW testing using structurally integrated piezos for SHM, and little or no theoretical basis is provided by researchers for their choice of the various testing parameters involved such as transducer geometry, dimensions, location and materials, excitation frequency and bandwidth among others. There have been a few efforts towards modeling of GW excitation and sensing using surface-bonded piezos. Moulin et al [14] used a coupled finite-element-normal mode expansion method to determine the amplitudes of the GW modes excited in a composite plate with surface-bonded/embedded piezos. The finite-element method was used in the area of the plate near the piezo, enabling the computation of the mechanical excitation field caused by the transducer, which was then introduced as a forcing function into the normal mode equations. This was also a $2 \mathrm{D}$ 'plane strain' analysis. Lin and Yuan [15] modeled the diagnostic transient waves in an infinite isotropic plate generated by a pair of surface-bonded circular actuators on either free surface at the same surface location excited out of phase with respect to each other. Mindlin plate theory incorporating transverse shear and rotary inertia effects was used and the actuators were modeled as causing bending moments along their edge. A simplified equation to describe the sensor response of a surface-bonded piezo-sensor was derived and some experimental verification for the model was provided. Rose and Wang [16] studied theoretical source solutions in isotropic plates, again using Mindlin plate theory, deriving expressions for the response to a point moment, point vertical force and various doublet combinations. These solutions were used to generate equations describing the displacement field patterns for circular and narrow rectangular piezo actuators, which were modeled as causing bending moments and moment doublets respectively along their edges. Veidt et al [17] used a hybrid theoretical-experimental approach for solving the excitation field due to surface-bonded rectangular and circular actuators. In the theoretical development, the piezoactuator was modeled as causing normal surface traction, and again Mindlin plate theory was used. The magnitude of the normal traction exerted for a certain frequency was estimated experimentally using a laser Doppler vibrometer, which was used to characterize the electromechanical transfer properties of the piezos. This hybrid approach was used to predict surface out-of-plane velocity signals with limited success. However, one major disadvantage of using Mindlin plate theory is that it can only approximately model the lowest antisymmetric $\left(\mathrm{A}_{0}\right)$ Lamb mode and it can only be used when the excitation frequency-plate thickness product is low enough that higher antisymmetric modes are not excited. In addition, it cannot model the symmetric Lamb modes or SH modes. Giurgiutiu [18] studied the harmonic excitation of Lamb waves in an isotropic plate to model the case of plane waves excited by infinitely wide surface-bonded piezos. As he suggested, the key difference between modeling NDE transducers and surface-bonded piezoelectric actuators is that the former operate by 'tapping' or causing normal traction on the surface, while the latter operate by 'pinching' or causing tangential traction at the actuator edges on the structure surface. The strain and displacement wave solutions were obtained using the Fourier integral transform applied to the 3D linear elasticity based Lamb-wave equations followed by inversion using residue theory, after they were simplified for the $2 \mathrm{D}$ nature of this problem.

In this work, first a generic procedure to solve for the GW fields excited by arbitrary-shaped piezo-actuators surfacebonded on an infinite isotropic plate is proposed. It is then used to generate solutions for the two particular transducer shapes that are most commonly used, namely ring-shaped and rectangular. The solution for circular actuators is presented as a special case of ring-shaped actuators and verified using finite-element method (FEM) simulations. A formulation is developed to obtain the response of piezo-sensors in GW fields. Experimental results to validate these solutions for two cases, i.e., circular and rectangular actuators, are discussed. Finally, these equations are used to find the optimal values of transducer dimensions. This work is unique in that rigorous analytical solutions based on $3 D$ linear elasticity are proposed for finite-dimensional actuators, and these are backed strongly by numerical and experimental results.

\section{GW excitation in a plate by an arbitrary shape surface-bonded piezo}

In this section, a general expression for the GW field excited by an arbitrary shape (finite-dimensional) piezo-actuator surfacebonded on an infinite isotropic plate is derived. This formulation is based on the $3 \mathrm{D}$ elasticity equations of motion. 


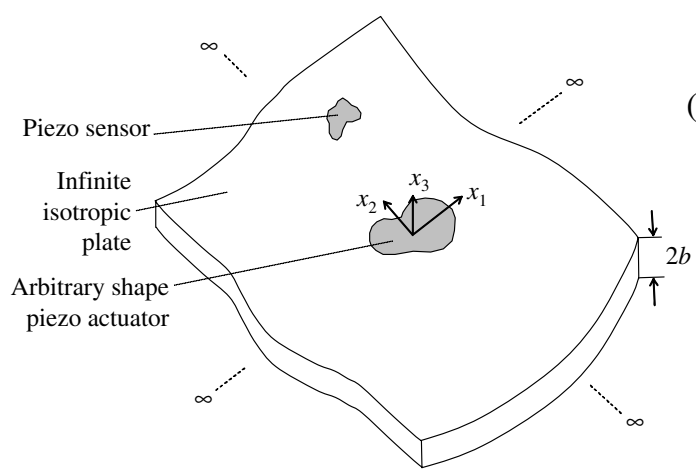

(1)

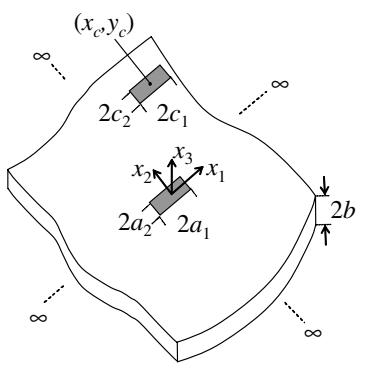

(2)

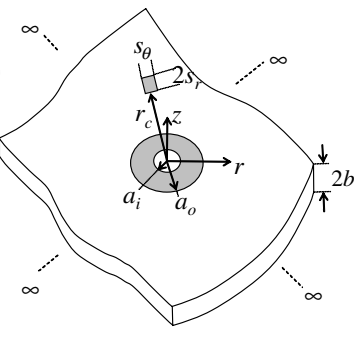

Figure 1. Infinite isotropic plate with arbitrary shape surface-bonded piezo-actuator and piezo-sensor and the two specific shapes considered.

Consider an infinite isotropic plate of thickness $2 b$ with such an actuator bonded on the surface $x_{3}=+b$, as illustrated in figure 1. The origin is located midway through the plate thickness and the $x_{3}$-axis is normal to the plate surface. The choice of in-plane location of the origin and the orientation of the axes $x_{1}$ and $x_{2}$ at this point is arbitrary, but it is relaxed later. The equations of motion are

$$
(\lambda+\mu) \nabla \nabla \cdot \boldsymbol{u}+\mu \nabla^{2} \boldsymbol{u}+\boldsymbol{f}=\rho \ddot{\boldsymbol{u}} .
$$

In this case the body force $f=0$. Furthermore, the equations of motion can be decomposed into the Helmholtz components using

$$
\begin{gathered}
\boldsymbol{u}=\nabla \phi+\nabla \times \mathbf{H} \\
\nabla \cdot \mathbf{H}=0 .
\end{gathered}
$$

It can be shown using equation (2) that equation (1) is equivalent to the equations

$$
\begin{array}{cc}
\nabla^{2} \phi=\frac{\ddot{\phi}}{c_{p}^{2}} ; & c_{p}^{2}=\frac{\lambda+2 \mu}{\rho} \\
\nabla^{2} \mathbf{H}=\frac{\ddot{\mathbf{H}}}{c_{s}^{2}} ; & c_{s}^{2}=\frac{\mu}{\rho} .
\end{array}
$$

Considering the response to harmonic excitation at angular frequency $\omega$, one obtains

$$
\frac{\partial^{2}()}{\partial t^{2}}=-\omega^{2}()
$$

The double spatial Fourier transform is used to ease solution of this problem. For a generic variable $\varphi$, it is defined by

$$
\bar{\varphi}\left(\xi_{1}, \xi_{2}\right)=\int_{-\infty}^{\infty} \int_{-\infty}^{\infty} \varphi\left(x_{1}, x_{2}\right) \mathrm{e}^{\mathrm{i}\left(\xi_{1} x_{1}+\xi_{2} x_{2}\right)} \mathrm{d} x_{1} \mathrm{~d} x_{2}
$$

and the inverse is given by

$$
\varphi\left(x_{1}, x_{2}\right)=\frac{1}{4 \pi^{2}} \int_{-\infty}^{\infty} \int_{-\infty}^{\infty} \bar{\varphi}\left(\xi_{1}, \xi_{2}\right) \mathrm{e}^{-\mathrm{i}\left(\xi_{1} x_{1}+\xi_{2} x_{2}\right)} \mathrm{d} \xi_{1} \mathrm{~d} \xi_{2} .
$$

Applying the double spatial Fourier transform on equations (3) and (4) and using equation (5), one obtains the following equations:

$$
\left(-\xi_{1}^{2}-\xi_{2}^{2}\right) \bar{\phi}+\frac{\mathrm{d}^{2} \bar{\phi}}{\mathrm{d} x_{3}^{2}}=-\frac{\omega^{2}}{c_{p}^{2}} \bar{\phi}
$$

$$
\left(-\xi_{1}^{2}-\xi_{2}^{2}\right) \overline{\mathbf{H}}+\frac{\mathrm{d}^{2} \overline{\mathbf{H}}}{\mathrm{d} x_{3}^{2}}=-\frac{\omega^{2}}{c_{s}^{2}} \overline{\mathbf{H}} .
$$

Let

$$
\left(-\xi_{1}^{2}-\xi_{2}^{2}\right)+\frac{\omega^{2}}{c_{p}^{2}}=\alpha^{2} ; \quad\left(-\xi_{1}^{2}-\xi_{2}^{2}\right)+\frac{\omega^{2}}{c_{s}^{2}}=\beta^{2} .
$$

The solutions of equations (8) and (9) are of the form (the $\mathrm{e}^{\mathrm{i} \omega t}$ factor is dropped from all subsequent equations and will be brought back in the final expression)

$$
\begin{aligned}
\bar{\phi} & =C_{1} \sin \alpha x_{3}+C_{2} \cos \alpha x_{3} \\
\overline{\mathrm{H}}_{1} & =C_{3} \sin \beta x_{3}+C_{4} \cos \beta x_{3} \\
\overline{\mathrm{H}}_{2} & =C_{5} \sin \beta x_{3}+C_{6} \cos \beta x_{3} \\
\overline{\mathrm{H}}_{3} & =C_{7} \sin \beta x_{3}+C_{8} \cos \beta x_{3} .
\end{aligned}
$$

Furthermore, it can be shown that the constants $C_{2}, C_{3}, C_{5}$ and $C_{8}$ are associated with symmetric modes and that the constants $C_{1}, C_{4}, C_{6}$ and $C_{7}$ are associated with antisymmetric modes. For the subsequent analysis, only the symmetric modes are considered. The contributions from antisymmetric modes can be derived analogously. The linear strain-displacement relation and the constitutive equations for linear elasticity yield

$$
\varepsilon_{i j}=\frac{1}{2}\left(u_{i, j}+u_{j, i}\right) ; \quad \sigma_{i j}=\lambda \varepsilon_{k k} \delta_{i j}+2 \mu \varepsilon_{i j} .
$$

Using equations (2), (11) and (12), it can be shown that the transformed stresses at $x_{3}=b$ are

$$
\begin{aligned}
& \bar{\sigma}_{33}=\mu\left[C_{2}\left(\xi_{1}^{2}+\xi_{2}^{2}-\beta^{2}\right) \cos \alpha b+C_{3}\left(2 \mathrm{i} \mu \xi_{2} \beta\right) \cos \beta b\right. \\
& \left.\quad+C_{5}\left(-2 \mathrm{i} \xi_{1} \beta\right) \cos \beta b\right] \\
& \bar{\sigma}_{32}=\mu\left[C_{2}\left(2 \mathrm{i} \xi_{2} \alpha\right) \sin \alpha b+C_{3}\left(\xi_{2}^{2}-\beta^{2}\right) \sin \beta b\right. \\
& \left.\quad+C_{5}\left(-\xi_{1} \xi_{2}\right) \sin \beta b+C_{8}\left(-\mathrm{i} \xi_{1} \beta\right) \sin \beta b\right] \\
& \bar{\sigma}_{31}=\mu\left[C_{2}\left(2 \mathrm{i} \alpha \xi_{1}\right) \sin \alpha b+C_{3}\left(\xi_{1} \xi_{2}\right) \sin \beta b\right. \\
& \left.\quad+C_{5}\left(\beta^{2}-\xi_{1}^{2}\right) \sin \beta b+C_{8}\left(\mathrm{i} \xi_{2} \beta\right) \sin \beta b\right] .
\end{aligned}
$$

The piezo-actuator is modeled as causing in-plane traction of uniform magnitude (say $\tau_{0}$ per unit length) along its perimeter, in the direction normal to the free edge on the plate surface $x_{3}=+b$. In this model, the dynamics of the actuator are neglected and it is assumed that the plate dynamics are 
uncoupled from the actuator dynamics. This model was proposed by Crawley and de Luis [19] to describe quasi-static induced strain actuation of piezo-actuators surface-bonded onto beams. For that case, they proved that the model is a good approximation if the product of the actuator Young's modulus and thickness is small compared to that of the substrate and the bond layer is thin and stiff. This is a practical assumption, since in the aerospace industry plate-like structures used are typically between 2 and $5 \mathrm{~mm}$ thick, while piezo-elements typically used are $0.2-0.5 \mathrm{~mm}$ thick. This assumption will be examined in further detail in section 7, where the experimental results are discussed. Another assumption made is that the piezoelectric properties of the piezo are constant over the frequency range of interest, which is supported by the work of González and Alemany [20]. In addition, material damping is neglected. This assumption is based on the fact that, for metals, attenuation from finite excitation sources dominates amplitude decay of the GW. The externally applied surface tangential traction components yield the following expressions for stresses at the free surface $x_{3}=+b$ and their double spatial Fourier transforms:

$$
\begin{aligned}
\sigma_{33}=0 ; & \bar{\sigma}_{33}=0 \\
\sigma_{32}=\tau_{0} \cdot F_{2}\left(x_{1}, x_{2}\right) ; & \bar{\sigma}_{32}=\tau_{0} \cdot \bar{F}_{2}\left(\xi_{1}, \xi_{2}\right) \\
\sigma_{31}=\tau_{0} \cdot F_{1}\left(x_{1}, x_{2}\right) ; & \bar{\sigma}_{31}=\tau_{0} \cdot \bar{F}_{1}\left(\xi_{1}, \xi_{2}\right)
\end{aligned}
$$

where $F_{1}$ and $F_{2}$ are arbitrary functions, that are zero everywhere except around the edge of the piezo-actuator. It would be prudent to choose the coordinate axes $x_{1}$ and $x_{2}$ as well as the origin's in-plane location to ease the computation of $\bar{F}_{1}$ and $\bar{F}_{2}$. Equating equations (13) and (14) would give three equations in four unknowns. The fourth equation results from the divergence condition on $\mathbf{H}$, and consequently $\overline{\mathbf{H}}$, given by

$$
\frac{\partial \overline{\mathrm{H}}_{1}}{\partial x_{1}}+\frac{\partial \overline{\mathrm{H}}_{2}}{\partial x_{2}}+\frac{\partial \overline{\mathrm{H}}_{3}}{\partial x_{3}}=0 .
$$

Using equations (11) in (15) and evaluating at $x_{3}=b$ gives

$$
C_{3}\left(-\mathrm{i} \xi_{1} \sin \beta b\right)+C_{5}\left(-\mathrm{i} \xi_{2} \sin \beta b\right)+C_{8}(-\beta \sin \beta b)=0 \text {. }
$$

With four equations and four unknowns, the unknown constants $C_{2}, C_{3}, C_{5}$ and $C_{8}$ can be solved for from the matrix equation:

$$
\begin{gathered}
{\left[\begin{array}{cc}
\left(\xi_{1}^{2}+\xi_{2}^{2}-\beta^{2}\right) \cos \alpha b & 2 \mathrm{i} \xi_{2} \beta \cos \beta b \\
2 \mathrm{i} \xi_{2} \alpha \sin \alpha b & \left(\xi_{2}^{2}-\beta^{2}\right) \sin \beta b \\
2 \mathrm{i} \xi_{1} \alpha \sin \alpha b & \xi_{1} \xi_{2} \sin \beta b \\
0 & -\mathrm{i} \xi_{1} \sin \beta b \\
-2 \mathrm{i} \xi_{1} \beta \cos \beta b & 0 \\
-\xi_{1} \xi_{2} \sin \beta b & -\mathrm{i} \xi_{1} \beta \sin \beta b \\
\left(\beta^{2}-\xi_{1}^{2}\right) \sin \beta b & \mathrm{i} \xi_{2} \beta \sin \beta b \\
-\mathrm{i} \xi_{2} \sin \beta b & -\beta \sin \beta b
\end{array}\right]\left[\begin{array}{c}
C_{2} \\
C_{3} \\
C_{5} \\
C_{8}
\end{array}\right]} \\
=\frac{\tau_{0}}{\mu}\left[\begin{array}{c}
0 \\
\bar{F}_{2}\left(\xi_{1}, \xi_{2}\right) \\
\bar{F}_{1}\left(\xi_{1}, \xi_{2}\right) \\
0
\end{array}\right] .
\end{gathered}
$$

Solving for the constants and applying the inverse Fourier transform ultimately yields the following expressions for the transformed displacement components on the free surface $x_{3}=b$ :

$$
\begin{aligned}
u_{1} & =\frac{\tau_{0}}{4 \pi^{2} \mu} \int_{-\infty}^{\infty} \int_{-\infty}^{\infty} \frac{\cos \beta b \cdot \mathrm{e}^{-\mathrm{i}\left(\xi_{1} x_{1}+\xi_{2} x_{2}-\omega t\right)}}{\beta \sin \beta b \cdot D_{S}(\xi)} \\
& \times\left\{-\bar{F}_{1}\left(\xi_{1}, \xi_{2}\right)\left[\left(\left(\xi_{2}^{2}-\beta^{2}\right)+\xi_{1}^{2}\left(\xi_{2}^{2}+\beta^{2}\right)\right) \cos \alpha b \sin \beta b\right.\right. \\
& \left.+4 \alpha \beta \xi_{2}^{2} \sin \alpha b \cos \beta b\right]+\bar{F}_{2}\left(\xi_{1}, \xi_{2}\right) \\
& \times\left[\xi_{1} \xi_{2}\left(\xi^{2}-3 \beta^{2}\right) \cos \alpha b \sin \beta b\right. \\
& \left.\left.+4 \alpha \beta \xi_{1} \xi_{2} \sin \alpha b \cos \beta b\right]\right\} \mathrm{d} \xi_{1} \mathrm{~d} \xi_{2} \\
u_{2} & =\frac{\tau_{0}}{4 \pi^{2} \mu} \int_{-\infty}^{\infty} \int_{-\infty}^{\infty} \frac{\cos \beta b \cdot \mathrm{e}^{-\mathrm{i}\left(\xi_{1} x_{1}+\xi_{2} x_{2}-\omega t\right)}}{\beta \sin \beta b \cdot D_{S}(\xi)}\left\{\bar{F}_{1}\left(\xi_{1}, \xi_{2}\right)\right. \\
& \times\left[\xi_{1} \xi_{2}\left(\xi^{2}-3 \beta^{2}\right) \cos \alpha b \sin \beta b+4 \alpha \beta \xi_{1} \xi_{2} \sin \alpha b \cos \beta b\right] \\
& -\bar{F}_{2}\left(\xi_{1}, \xi_{2}\right)\left[\left(\left(\xi_{1}^{2}-\beta^{2}\right)^{2}+\xi_{2}^{2}\left(\xi_{1}^{2}+\beta^{2}\right)\right) \cos \alpha b \sin \beta b\right. \\
& \left.\left.+4 \alpha \beta \xi_{1}^{2} \sin \alpha b \cos \beta b\right]\right\} \mathrm{d} \xi_{1} \mathrm{~d} \xi_{2} \\
u_{3} & =\frac{\tau_{0}}{4 \pi^{2} \mu} \int_{-\infty}^{\infty} \int_{-\infty}^{\infty} \frac{-\mathrm{ie}{ }^{-i\left(\xi_{1} x_{1}+\xi_{2} x_{2}-\omega t\right)}}{D_{S}\left(\xi_{)}\right)} \\
& \times\left[2 \alpha \beta \sin \alpha b \cos \beta b+\left(\xi^{2}-\beta^{2}\right) \sin \beta b \cos \alpha b\right] \\
& \times\left(\xi_{1} \bar{F}_{1}\left(\xi_{1}, \xi_{2}\right)+\xi_{2} \bar{F}_{2}\left(\xi_{1}, \xi_{2}\right)\right) \mathrm{d} \xi_{1} \mathrm{~d} \xi_{2}
\end{aligned}
$$

where $\xi^{2}=\xi_{1}^{2}+\xi_{2}^{2}$ and $D_{S}(\xi)=\left(\xi^{2}-\beta^{2}\right)^{2} \cos \alpha b \sin \beta b+$ $4 \xi^{2} \alpha \beta \sin \alpha b \cos \beta b$. These integrals could be singular at the points corresponding to the real roots of either $D_{S}=0$ or $\sin \beta b=0$ or both (depending on which term(s) survive after substituting $\bar{F}_{1}$ and $\bar{F}_{2}$ ). The former correspond to the wavenumbers, $\xi^{S}$, from the solutions of the RayleighLamb equation for symmetric modes at frequency $\omega$. The latter correspond to the wavenumbers of horizontally polarized symmetric mode shear ( $\mathrm{SH})$ waves, also at frequency $\omega$. In principle, one can also include the contributions from the imaginary and complex wavenumbers satisfying these equations. However, these are usually not of interest, since they yield evanescent or standing waves that decay very rapidly away from the source. While in the above derivation only symmetric modes were considered, the contribution from antisymmetric modes can be found analogously and the final solution would be a superposition of these two modal contributions. The inversion of these integrals is presented for two specific configurations of interest in the next section.

\section{Particular configurations}

In this section, the general derivation from section 2 is used to solve for the harmonic GW field due to two particular shapes of piezo-actuators commonly used in GW SHM, namely a rectangular piezo and a ring-shaped piezo. These two cases are labeled (1) and (2) respectively and the coordinate system and dimensions for each case are as illustrated in figure 1 .

\subsection{Rectangular piezo}

In this case, the functions $F_{1}$ and $F_{2}$ and their respective Fourier transforms are

$$
\begin{gathered}
F_{1}=\left[\delta\left(x_{1}-a_{1}\right)-\delta\left(x_{1}+a_{1}\right)\right]\left[H e\left(x_{2}+a_{2}\right)-H e\left(x_{2}-a_{2}\right)\right] \\
\bar{F}_{1}=-4 \sin \left(\xi_{1} a_{1}\right) \sin \left(\xi_{2} a_{2}\right) / \mathrm{i} \xi_{2} \\
F_{2}=\left[H e\left(x_{1}+a_{1}\right)-H e\left(x_{1}-a_{1}\right)\right]\left[\delta\left(x_{2}-a_{2}\right)-\delta\left(x_{2}+a_{2}\right)\right] \\
\bar{F}_{2}=-4 \sin \left(\xi_{1} a_{1}\right) \sin \left(\xi_{2} a_{2}\right) / \mathrm{i} \xi_{1} .
\end{gathered}
$$


Substituting equations (21) in equation (18) ultimately gives the following expression for displacement along the 1direction:

$$
\begin{aligned}
& u_{1}^{S}\left(x_{3}=b\right)=\frac{\mathrm{e}^{\mathrm{i} \omega t}}{4 \pi^{2}} \int_{-\infty}^{\infty} \int_{-\infty}^{\infty} \frac{4 \tau_{0} \sin \xi_{1} a_{1} \sin \xi_{2} a_{2}}{\mathrm{i} \mu \xi_{2} \xi} \\
& \times \frac{N_{S}(\xi)}{D_{S}(\xi)} \mathrm{e}^{-\mathrm{i}\left(\xi_{1} x_{1}+\xi_{2} x_{2}\right)} \mathrm{d} \xi_{1} \mathrm{~d} \xi_{2}
\end{aligned}
$$

where $N_{S}(\xi)=\xi \beta\left(\xi^{2}+\beta^{2}\right) \cos \alpha b \cos \beta b$. Observe that the $\sin \beta b$ term is absent in the denominator here, implying that only Lamb waves are excited in this case. Transforming into polar coordinates gives

$$
\begin{aligned}
& u_{1}^{S}\left(x_{3}=b\right)=\frac{\tau_{0} \mathrm{e}^{\mathrm{i} \omega t}}{\pi^{2} \mathrm{i} \mu} \int_{0}^{\infty} \int_{0}^{2 \pi} \frac{\sin \left(\xi \cos \gamma a_{1}\right) \sin \left(\xi \sin \gamma a_{2}\right)}{\xi^{2} \sin \gamma} \\
& \times \frac{N_{S}(\xi)}{D_{S}(\xi)} \mathrm{e}^{-\mathrm{i} \xi\left(x_{1} \cos \gamma+x_{2} \sin \gamma\right)} \xi \mathrm{d} \gamma \mathrm{d} \xi .
\end{aligned}
$$

The integral in the real $\xi-\gamma$ plane is replaced by a surface integral in the complex $\xi-\gamma$ space. The values of $x_{1}$ and $x_{2}$ will determine the shape of the surface. For example, if $x_{1}>$ $a_{1}, x_{2}>a_{2}$, then contributions from negative wavenumbers are not allowed on physical grounds, hence the integral must only include the first quadrant, i.e., $\gamma \in(0, \pi / 2)$ and the lower half of the complex $\xi-\gamma$ space as shown in figure 2. The integrand is singular at the roots of $D_{S}=0$, designated $\xi^{S}$. Using the residue theorem yields in this case:

$$
\begin{aligned}
\int_{0}^{\infty} \int_{0}^{2 \pi} I \mathrm{~d} \gamma \mathrm{d} \xi+\int_{C} I \mathrm{~d} \gamma \mathrm{d} \xi & \operatorname{Res}\left(I\left(\xi^{S}\right)\right) \mathrm{d} \gamma \quad \text { where } \\
& =-\pi \mathrm{i} \sum_{\xi^{S}} \int_{0}^{\pi / 2}{\sin \left(\xi \cos \gamma a_{1}\right) \sin \left(\xi \sin \gamma a_{2}\right)}_{\xi \sin \gamma} \frac{N_{S}(\xi)}{D_{S}(\xi)} \mathrm{e}^{-\mathrm{i} \xi\left(x_{1} \cos \gamma+x_{2} \sin \gamma\right)}
\end{aligned}
$$

where $C$ is the semi-spherical surface in the lower half-plane, while 'Res' stands for the residue of the integrand at the singularities of $I$. The contribution from $C$ vanishes as the radius of the surface $R \rightarrow \infty$, as explained in [21] for a similar plane-wave excitation problem. Thus, the following expression is obtained for displacement in the region $x_{1}>$ $a_{1}, x_{2}>a_{2}$ :

$$
\begin{aligned}
& u_{1}^{S}\left(x_{3}=b\right)=\sum_{\xi^{S}} \frac{-\tau_{0}}{\pi \mu} \frac{N_{S}\left(\xi^{S}\right)}{\xi^{S} D_{S}^{\prime}\left(\xi^{S}\right)} \mathrm{e}^{\mathrm{i} \omega t} \\
& \quad \times \int_{0}^{\pi / 2} \frac{\sin \left(\xi^{S} a_{1} \cos \gamma\right) \sin \left(\xi^{S} a_{2} \sin \gamma\right)}{\sin \gamma} \\
& \quad \times \mathrm{e}^{-\mathrm{i} \xi^{S}\left(x_{1} \cos \gamma+x_{2} \sin \gamma\right)} \mathrm{d} \gamma .
\end{aligned}
$$

An approximate closed form solution can be obtained for the far field using the method of stationary phase. As explained in Graff [22], for large $r$

$$
\int_{\psi_{1}}^{\psi_{2}} f(\psi) \mathrm{e}^{\mathrm{i} r h(\psi)} \mathrm{d} \psi=\sqrt{\frac{2 \pi}{r h^{\prime \prime}\left(\psi_{0}\right)}} f\left(\psi_{0}\right) \mathrm{e}^{\mathrm{i}\left(r \psi_{0}+\pi / 4\right)}
$$

where $h^{\prime}\left(\psi_{0}\right)=0, f()$ is an arbitrary function, and $\psi_{1}$ and $\psi_{2}$ are arbitrary end-points of the interval of integration, which contains $\psi_{0}$. Hence, the following asymptotic expression

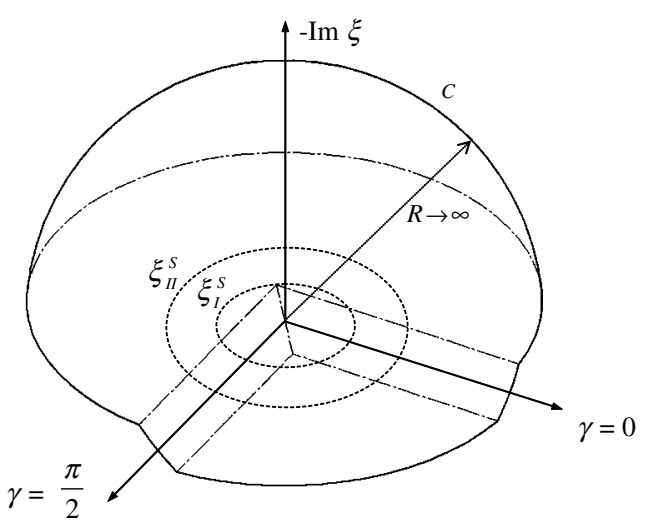

Figure 2. Contour integral in the complex $\xi$-plane used to invert the displacement integral using residue theory.

holds for the particle displacement in the far field in the region $x_{1}>a_{1}, x_{2}>a_{2}$ :

$$
\begin{aligned}
& u_{1}^{S}\left(x_{3}=b\right)=\sum_{\xi^{S}} \frac{-\tau_{0}}{\pi \mu} \frac{N_{S}\left(\xi^{S}\right)}{\xi^{S} D_{S}^{\prime}\left(\xi^{S}\right)} \sqrt{\frac{2 \pi}{\xi^{S} r}} \\
& \times \frac{\sin \left(\xi^{S} a_{1} \cos \theta\right) \sin \left(\xi^{S} a_{2} \sin \theta\right)}{\sin \theta} \mathrm{e}^{-\mathrm{i}\left(\xi^{S} r+\pi / 4-\omega t\right)}
\end{aligned}
$$

where $\theta=\tan ^{-1}\left(x_{2} / x_{1}\right)$ and $r=\sqrt{x_{1}^{2}+x_{2}^{2}}$. This indicates that the GW field tends to a circular crested field with angularly dependent amplitude at large distances from the actuator. In other regions of the plate, the region of the contour included will change. For example, in the region $x_{1}>a_{1},-a_{2}<x_{2}<a_{2}$, due to the presence of both positive and negative wavenumbers along the $x_{2}$-direction and only positive wavenumbers along the $x_{1}$-direction, contributions to the integral over the range $\gamma=-\pi / 2$ to $\pi / 2$ must be included. Thus for this region the expression changes to

$$
\begin{aligned}
& u_{1}^{S}\left(x_{3}=b\right)=\sum_{\xi^{S}} \frac{-\tau_{0}}{\pi \mu} \frac{N_{S}\left(\xi^{S}\right)}{\xi^{S} D_{S}^{\prime}\left(\xi^{S}\right)} \\
& \times \mathrm{e}^{\mathrm{i} \omega t} \int_{-\pi / 2}^{\pi / 2} \frac{\sin \left(\xi^{S} a_{1} \cos \gamma\right) \sin \left(\xi^{S} a_{2} \sin \gamma\right)}{\sin \gamma} \\
& \times \mathrm{e}^{-\mathrm{i} \xi^{S}\left(x_{1} \cos \gamma+x_{2} \sin \gamma\right)} \mathrm{d} \gamma
\end{aligned}
$$

which can be shown to be equivalent to

$$
\begin{aligned}
& u_{1}^{S}\left(x_{3}=b\right)=\sum_{\xi^{S}} \frac{-2 \tau_{0}}{\pi \mu} \frac{N_{S}\left(\xi^{S}\right)}{\xi^{S} D_{S}^{\prime}\left(\xi^{S}\right)} \\
& \times \mathrm{e}^{\mathrm{i} \omega t} \int_{0}^{\pi / 2} \frac{\sin \left(\xi^{S} a_{1} \cos \gamma\right) \sin \left(\xi^{S} a_{2} \sin \gamma\right)}{\sin \gamma} \\
& \times \cos \left(\xi^{S} x_{2} \sin \gamma\right) \mathrm{e}^{-\mathrm{i} \xi^{S} x_{1} \cos \gamma} \mathrm{d} \gamma .
\end{aligned}
$$

Similarly, expressions for the other displacement components can be obtained. 

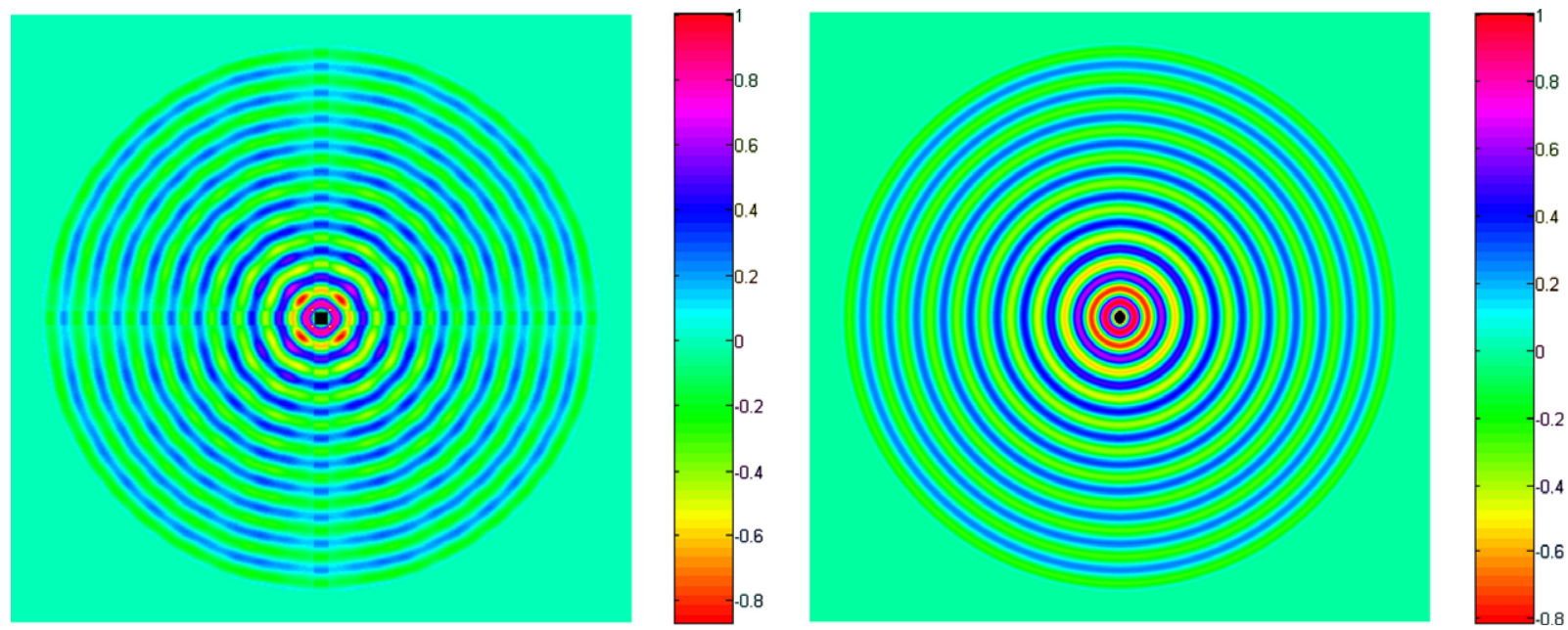

Figure 3. Harmonic radiation field for out-of-plane surface displacement $\left(u_{3}\right)$ in a $2 \mathrm{~mm}$ thick aluminum plate at $100 \mathrm{kHz}, \mathrm{A}_{0}$ mode, by a pair of (a) (left) $0.5 \mathrm{~cm} \times 0.5 \mathrm{~cm}$ square actuators and (b) (right) $0.5 \mathrm{~cm}$ diameter circular actuators (in black, center) (normalized scales).

\subsection{Ring-shaped piezo}

In this case, the functions $F_{1}$ and $F_{2}$ and their respective Fourier transforms are

$$
\begin{gathered}
F_{1}=\left[\delta\left(r-a_{o}\right)-\delta\left(r-a_{i}\right)\right] \cos \theta \\
\bar{F}_{1}=-\mathrm{i}\left(a_{o} J_{1}\left(\xi a_{o}\right)-a_{i} J_{1}\left(\xi a_{i}\right)\right) \xi_{1} / \xi \\
F_{2}=\left[\delta\left(r-a_{o}\right)-\delta\left(r-a_{i}\right)\right] \sin \theta \\
\bar{F}_{2}=-\mathrm{i}\left(a_{o} J_{1}\left(\xi a_{o}\right)-a_{i} J_{1}\left(\xi a_{i}\right)\right) \xi_{2} / \xi
\end{gathered}
$$

where $J_{1}()$ is the Bessel function of the first kind and order unity. Using equations (18) and (30), one can show that

$$
\begin{aligned}
& u_{1}^{S}\left(x_{3}=b\right)=\frac{-\mathrm{i} \tau_{0}}{4 \pi^{2} \mu} \mathrm{e}^{\mathrm{i} \omega t} \int_{-\infty}^{\infty} \int_{-\infty}^{\infty}\left(a_{o} J_{1}\left(\xi^{S} a_{o}\right)-a_{i} J_{1}\left(\xi^{S} a_{i}\right)\right) \\
& \times \frac{N_{S}\left(\xi^{S}\right)}{\xi D_{S}\left(\xi^{S}\right)} \mathrm{e}^{-\mathrm{i}\left(\xi_{1} x_{1}+\xi_{2} x_{2}\right)} \mathrm{d} \xi_{1} \mathrm{~d} \xi_{2}
\end{aligned}
$$

where $N_{S}$ is as defined in section 3.1. Observe that in this case too only Lamb waves are excited. As before, only the symmetric modes are being considered here. Transforming into polar coordinates yields

$$
\begin{aligned}
& u_{1}^{S}\left(x_{3}=b\right)=\frac{-\mathrm{i} \tau_{0}}{4 \pi^{2} \mu} \mathrm{e}^{\mathrm{i} \omega t} \int_{0}^{\infty} \int_{0}^{2 \pi}\left(a_{o} J_{1}\left(\xi a_{o}\right)-a_{i} J_{1}\left(\xi a_{i}\right)\right) \\
& \times \frac{N_{S}(\xi)}{D_{S}(\xi)} \mathrm{e}^{-\mathrm{i} \xi\left(x_{1} \cos \gamma+x_{2} \sin \gamma\right)} \cos \gamma \mathrm{d} \gamma \mathrm{d} \xi .
\end{aligned}
$$

Without loss of generality due to axisymmetry, consider the point $x_{1}=r, x_{2}=0$. Only the solution outside the excitation region, i.e. $r>a_{o}$, is of interest. A similar approach as in section 3.1 is used to invert the integral. The integration contour in the complex $\xi$-space used is also similar to the one in figure 2. However, in this case on physical grounds, contributions from negative wavenumbers along the $x_{1}$-direction are not allowed, hence the range from $\gamma=-\pi / 2$ to $\pi / 2$ needs be considered. Hence, the expression for displacement becomes

$$
u_{1}^{S}\left(x_{3}=b\right)=\frac{-\pi \tau_{0}}{4 \pi \mu} \mathrm{e}^{\mathrm{i} \omega t} \sum_{\xi^{S}} \int_{-\pi / 2}^{\pi / 2}\left(a_{o} J_{1}\left(\xi a_{o}\right)-a_{i} J_{1}\left(\xi a_{i}\right)\right)
$$

$$
\begin{aligned}
& \times \frac{N_{S}(\xi)}{D_{S}^{\prime}(\xi)} \mathrm{e}^{-\mathrm{i}(\xi r \cos \gamma)} \cos \gamma \mathrm{d} \gamma \\
= & \frac{\pi \mathrm{i} \tau_{0}}{4 \mu} \mathrm{e}^{\mathrm{i} \omega t} \sum_{\xi^{S}}\left(a_{o} J_{1}\left(\xi a_{o}\right)-a_{i} J_{1}\left(\xi a_{i}\right)\right) \frac{N_{S}(\xi)}{D_{S}^{\prime}(\xi)} H_{1}^{(2)}(\xi r)
\end{aligned}
$$

where $H_{1}^{(2)}()$ is the complex Hankel function of order unity and the second type, defined by

$$
H_{1}^{(2)}(\xi r)=J_{1}(\xi r)-\mathrm{i} Y_{1}(\xi r)
$$

where $Y_{1}()$ is the Bessel function of the second kind of order unity. Similarly, expressions for the other displacement components can be obtained. In particular, it will be found that

$$
u_{2}^{S}\left(x_{1}=r, x_{2}=0\right)=0 .
$$

And since the point $(r, 0)$ is generic, by axisymmetry, equation (33) also represents the radial displacement at a point at distance $r$ from the center. The angular displacement is zero at all points. The solution for a circular actuator can be recovered simply by letting $a_{i}=0$ in the above equations. In addition, the following asymptotic expression holds for the complex Hankel function:

$$
\lim _{\xi r \rightarrow \infty} H_{1}^{(2)}(\xi r)=-\sqrt{\frac{1}{\pi \xi r}}(1+\mathrm{i}) \mathrm{e}^{-\mathrm{i} \xi r} .
$$

In practice, the Hankel function is very close to its asymptotic expression after the first four or five spatial wavelengths. Thus, the solution for a circular-crested Lamb-wave field tends to that of a spatially decaying plane Lamb-wave field after a few spatial oscillations.

The harmonic out-of-plane displacement patterns due to excitation of the $\mathrm{A}_{0}$ Lamb mode at $100 \mathrm{kHz}$ in a $2 \mathrm{~mm}$ aluminum plate by rectangular and circular actuators are shown in figure 3. For ease of visualization, only $10 \mathrm{~cm} \times 10 \mathrm{~cm}$ of the plate is shown and the field was set to zero for radius $r>9 \mathrm{~cm}$ in both cases. Figure 3(a) illustrates how the GW field due to a rectangular actuator tends to a circular crested GW field 

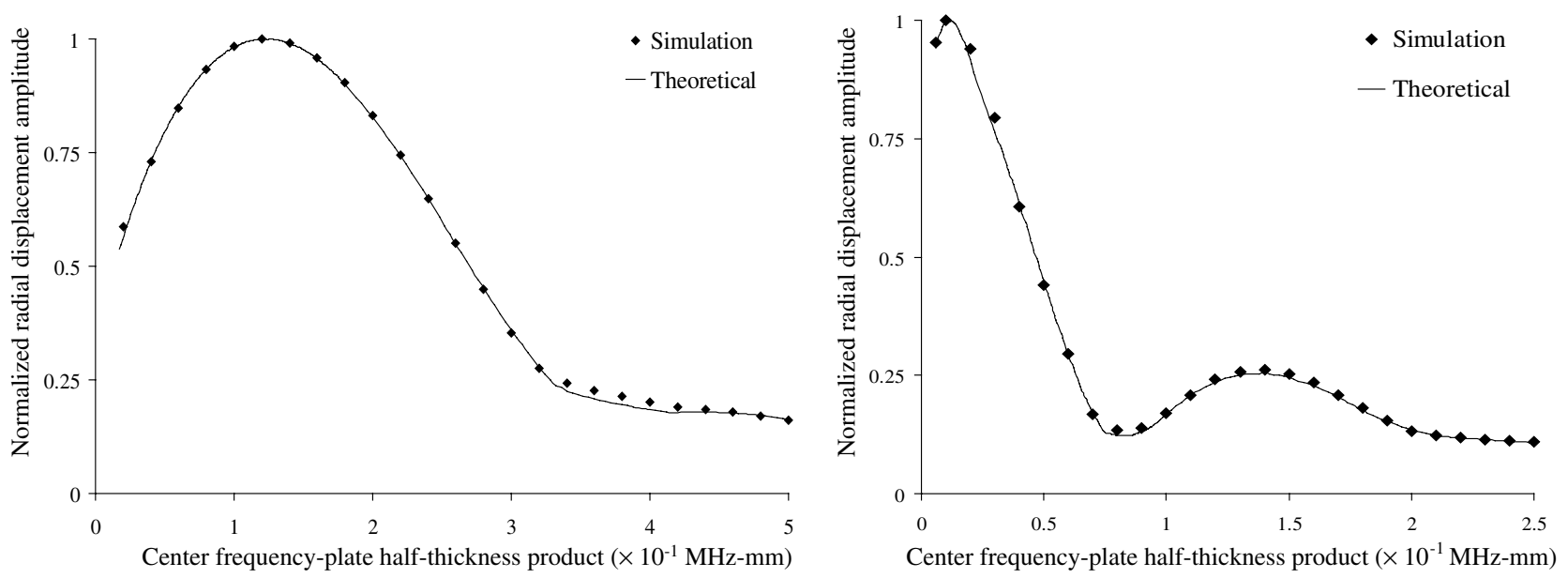

Figure 4. Comparison of proposed theoretical and FEM simulation results for the normalized radial displacement at $r=5 \mathrm{~cm}$ over various frequencies for $\mathrm{S}_{0}$ (left) and $\mathrm{A}_{0}$ (right) modes.

with angularly dependent amplitude at large distances from the actuator. The circular actuator GW field spatially decays with equally spaced peaks and troughs in the far field (see figure 3(b)).

While in this analysis it was assumed that a single angular frequency $\omega$ was excited, it can be used to find the response to any time-limited signal. This can be accomplished by taking the inverse Fourier transform of the integral of the product of the harmonic response multiplied by the Fourier transform of the excitation signal over the bandwidth.

\section{Verification of the circular actuator model}

In order to verify the result of the formulation proposed in section 3, FEM simulations were conducted using ABAQUS [23]. An infinite isotropic (aluminum alloy) plate with a $0.9 \mathrm{~cm}$ radius piezo-actuator placed at the origin of the coordinate system was modeled using a mesh of axisymmetric four-node continuum finite elements up to radial position $r=15 \mathrm{~cm}$. These were radially followed by infinite axisymmetric elements placed at $r=15 \mathrm{~cm}$, which are used to minimize the reflected waves returning from the boundary at $r=15 \mathrm{~cm}$ towards the origin. The FEM model represented only half the plate thickness, and then a throughthickness symmetry or antisymmetry condition was applied to the mid-thickness nodes to model symmetric or antisymmetric modes, respectively. The actuator was modeled as causing a surface radial shear force at $r=0.9 \mathrm{~cm}$, just as in the proposed formulation. A 3.5-cycle Hann window modulated sinusoidal toneburst excitation signal applied to the actuator was modeled by specifying the corresponding waveform for the time variation of the shear force applied at $r=0.9 \mathrm{~cm}$ in the input file. The amplitude of radial displacement at $r=5 \mathrm{~cm}$ was recorded for a range of values of the toneburst center frequency-plate thickness product. The mesh density and the time step were chosen to be sufficiently small to resolve the smallest wavelength and capture the highest frequency response, respectively. Two sets of simulations were performed: for symmetric and for antisymmetric modes. These were compared with the analytical predictions by the proposed formulation in section 3.2 (while considering the frequency bandwidth excited). The results are shown in figure 4. The FEM results compare very well with the theoretical predictions for both the $\mathrm{S}_{0}$ and $\mathrm{A}_{0}$ modes, providing verification for the proposed analytical formulation.

\section{Piezo-sensor response derivation}

In this section, the response of a piezo-sensor operating in the 3-1 mode and surface-bonded on a plate in a GW field is derived. The relation between the electric field $E_{i}$, displacement $D_{i}$ and internal stress in the piezoelectric element is [24]

$$
E_{i}=-g_{i k l} \sigma_{k l}+\beta_{i k}^{\sigma} D_{k}
$$

where $g_{i k l}$ is a matrix of piezoelectric constants for the piezoelectric material, and $\beta_{i k}^{\sigma}$ are the impermittivity constants at constant stress of the piezoelectric material. Since there is no external driving field, $E_{3}=0$, and since the sensor is poled in the 3-direction, $D_{1}=D_{2}=0$. Furthermore, if the sensor is thin enough, $\sigma_{33} \approx 0$. In addition, $\beta_{33}^{\sigma}=1 / k_{c} \varepsilon_{0}$. Thus, one obtains

$$
D_{3}=k_{c} \varepsilon_{0} g_{31}\left(\sigma_{i i}\right)=\frac{k_{c} \varepsilon_{0} g_{31} Y_{c}^{11}}{1-v_{c}}\left(\varepsilon_{i i}\right)
$$

where $Y_{c}^{11}$ is the in-plane Young's modulus of the sensor material, $v_{c}$ is the Poisson ratio of the piezoelectric material, $k_{c}$ is the dielectric constant of the sensor material, $\varepsilon_{0}$ is the permittivity of vacuum, and $\varepsilon_{i i}$ is the sum of the in-plane normal surface strains. Note that the contracted notation has been used for the $g$-constant indices from equation (38) onwards. Here it is assumed that the twisting shear stresses are negligible. The total electrical charge accumulated on the piezo-sensor's electrode surface is

$$
Q_{c}=\int_{\mathrm{S}_{c}}\left(D_{i} n_{i}\right) \mathrm{d} S=\int_{\mathrm{S}_{c}} D_{3} \mathrm{~d} S=\frac{k \varepsilon_{0} Y_{c}^{11} g_{31}}{1-v_{c}} \int_{\mathrm{S}_{c}} \varepsilon_{i i} \mathrm{~d} S
$$

where $\mathrm{S}_{c}$ is the surface area of the sensor, and $n_{i}$ are the components of the unit normal to the electrode surface of 
Finite-dimensional piezoelectric transducer modeling for guided wave based structural health monitoring
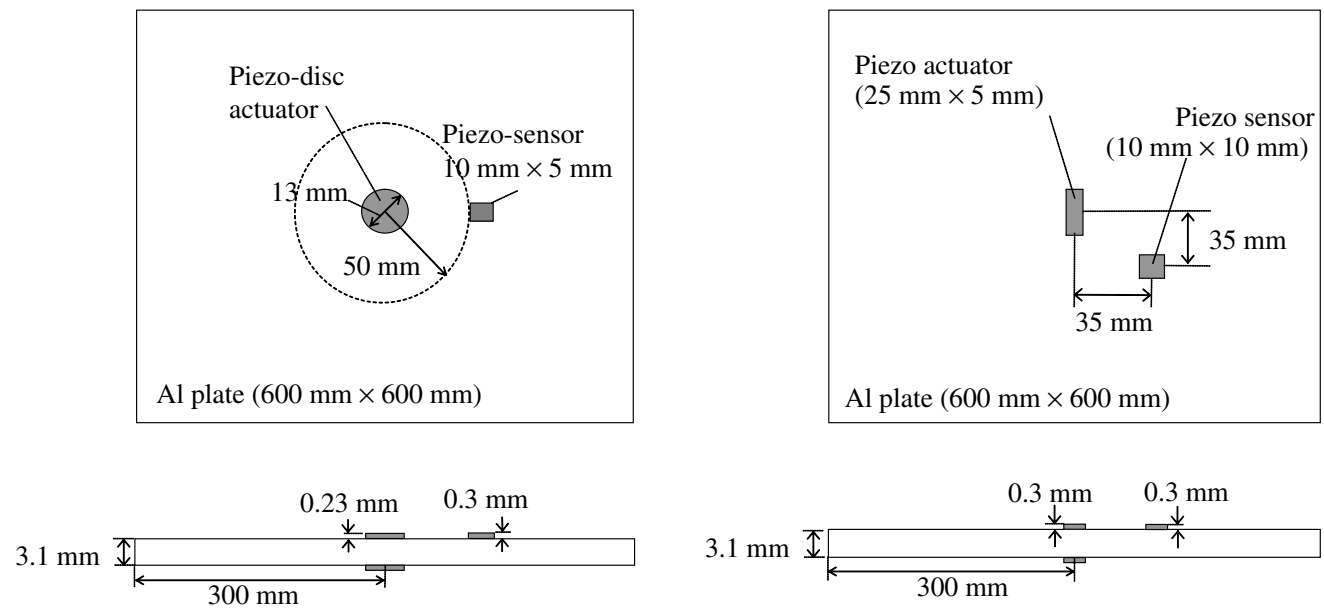

Figure 5. Experimental set-ups for validation of (a) circular actuator model (left) and (b) rectangular actuator model (right)

the piezo-sensor. In this case, $\left(n_{1}, n_{2}, n_{3}\right)=(0,0,1)$. An important assumption made here is that the sensor is infinitely compliant and does not disturb the GW field. This is reasonably satisfied if the product of the sensor's thickness and Young's modulus is small compared to that of the plate on which it is surface-bonded and it is of small size. The capacitance of the sensor is given by

$$
C_{c}=\frac{k_{c} \varepsilon_{0} \mathrm{~S}_{c}}{h_{c}}
$$

where $h_{c}$ is the sensor thickness. By treating the piezo-sensor as a capacitor, the output voltage response of the piezo-sensor is obtained as

$$
V_{c}=\frac{Q_{c}}{C_{c}}=\frac{Y_{c}^{11} h_{c} g_{31}}{\mathrm{~S}_{c}\left(1-v_{c}\right)} \int_{\mathrm{S}_{c}} \varepsilon_{i i} \mathrm{~d} S .
$$

Equation (41) is used in the next two sub-sections to evaluate the response of a piezo-sensor in GW fields due to circular and rectangular piezo-actuators.

\subsection{Piezo-sensor response in GW fields due to circular piezo-disc actuators}

Consider the response to harmonic excitation of a rectangular piezo-sensor of width $s_{\theta}$ in a circular-crested GW field placed between $r=r_{c}$ and $r=r_{c}+2 s_{r}$, as illustrated in figure 1 (with $a_{i}=0$ and $a_{o}=a$ ). In this case, equation (41) becomes

$$
\begin{aligned}
V_{c} & =\frac{Y_{c}^{11} h_{c} g_{31}}{\mathrm{~S}_{c}\left(1-v_{c}\right)} \int_{\mathrm{S}_{c}} \int\left(\varepsilon_{r r}+\varepsilon_{\theta \theta}\right) r \mathrm{~d} r \mathrm{~d} \theta \\
& =\frac{Y_{c}^{11} h_{c} g_{31}}{\mathrm{~S}_{c}\left(1-v_{c}\right)} \int_{\mathrm{S}_{c}} \int\left(\frac{\mathrm{d} u_{\mathrm{r}}}{\mathrm{d} r}+\frac{u_{\mathrm{r}}}{r}\right) r \mathrm{~d} r \mathrm{~d} \theta .
\end{aligned}
$$

Suppose that the width of the piezo-sensor $s_{\theta}$ is small enough so that $\int_{\theta} r \mathrm{~d} \theta \approx s_{\theta}$ over the radial length of the sensor. Using this and equations (33) and (42), one obtains (for symmetric modes)

$$
\begin{aligned}
V_{c}^{S} & =\frac{\mathrm{i} \tau_{0} a}{4 \mu} \frac{Y_{c}^{11} h_{c} g_{31}}{2 s_{r}\left(1-v_{c}\right)} \mathrm{e}^{\mathrm{i} \omega t} \sum_{\xi S} J_{1}\left(\xi^{S} a\right) \\
& \times \frac{N_{S}\left(\xi^{S}\right)}{D_{S}^{\prime}\left(\xi^{S}\right)} \int_{r_{c}}^{r_{c}+2 S_{\mathrm{r}}} \xi^{S} H_{0}^{(2)}\left(\xi^{S} r\right) \mathrm{d} r .
\end{aligned}
$$

\subsection{Piezo-sensor response in $G W$ fields due to rectangular piezo-actuators}

Consider the response to harmonic excitation of a rectangular piezo-sensor placed between the coordinates $\left(x_{c}-s_{1}, y_{c}-s_{2}\right)$ and $\left(x_{c}+s_{1}, y_{c}+s_{2}\right)$ with its edges along the $x_{1}$ and $x_{2}$ directions in the GW field due to a rectangular piezo-actuator described in section 3, as illustrated in figure 1. It is assumed that the sensor lies in the region $x_{1}>a_{1}, x_{2}>a_{2}$. In this case, equation (41) becomes

$$
\begin{aligned}
V_{c} & =\frac{Y_{c}^{11} h_{c} g_{31}}{4 s_{1} s_{2}\left(1-v_{c}\right)} \int_{\mathrm{S}_{c}} \int\left(\varepsilon_{11}+\varepsilon_{22}\right) \mathrm{dS}_{c} \\
& =\frac{Y_{c}^{11} h_{c} g_{31}}{4 s_{1} s_{2}\left(1-v_{c}\right)} \int_{x_{c}-s_{1}}^{x_{c}+s_{1}} \int_{y_{c}-s_{2}}^{y_{c}+s_{2}}\left[\frac{\mathrm{d} u_{1}}{\mathrm{~d} x_{1}}+\frac{\mathrm{d} u_{2}}{\mathrm{~d} x_{2}}\right] \mathrm{d} x_{1} \mathrm{~d} x_{2} .
\end{aligned}
$$

Using equation (25) and its analogue for $u_{2}$ along with equation (41) (for symmetric modes) yields

$$
\begin{aligned}
V_{c}^{S} & =\sum_{\xi^{S}} \frac{-8 \tau_{0} Y_{c}^{11} h_{c} g_{31}}{\pi \mu s_{1} s_{2}\left(1-v_{c}\right)} \frac{N_{S}\left(\xi^{S}\right)}{\left(\xi^{S}\right)^{2} D_{S}^{\prime}\left(\xi^{S}\right)} \mathrm{e}^{\mathrm{i} \omega t} \\
& \times \int_{0}^{\frac{\pi}{2}}\left\{\left[\sin \left(\xi^{S} a_{1} \cos \gamma\right) \sin \left(\xi^{S} a_{2} \sin \gamma\right) \sin \left(\xi^{S} s_{1} \cos \gamma\right)\right.\right. \\
& \left.\left.\times \sin \left(\xi^{S} S_{2} \sin \gamma\right)\right]\left[\sin ^{2} 2 \gamma\right]^{-1}\right\} \mathrm{e}^{-\mathrm{i} \xi^{S}\left(x_{c} \cos \gamma+y_{c} \sin \gamma\right)} \mathrm{d} \gamma .
\end{aligned}
$$

\section{Experimental set-ups and results}

To examine the validity of the theoretical expressions for the circular actuator derivation, the following experiment was performed. A $600 \mathrm{~mm} \times 600 \mathrm{~mm} \times 3.1 \mathrm{~mm}$ thick aluminum alloy plate (Young's modulus $Y_{\mathrm{Al}}=70.28 \mathrm{GPa}$, Poisson's ratio $v=0.33$, density $\rho=2684 \mathrm{~kg} \mathrm{~m}^{-3}$ ) was instrumented with a pair of $6.5 \mathrm{~mm}$ radius, $0.23 \mathrm{~mm}$ thick PZT-5H circular piezodisc actuators at the center of the plate on both free surfaces. A $10 \mathrm{~mm}$ (radial length) $\times 5 \mathrm{~mm}$ (width) $\times 0.3 \mathrm{~mm}$ (thickness) PZT-5A rectangular piezo-sensor was surface-bonded at a radial distance $r_{c}=50 \mathrm{~mm}$ relative to the center of the plate, as illustrated in figure 5(a). The set-up was designed such that reflections from the boundaries would not interfere with the first transmitted pulse received by the sensor over 

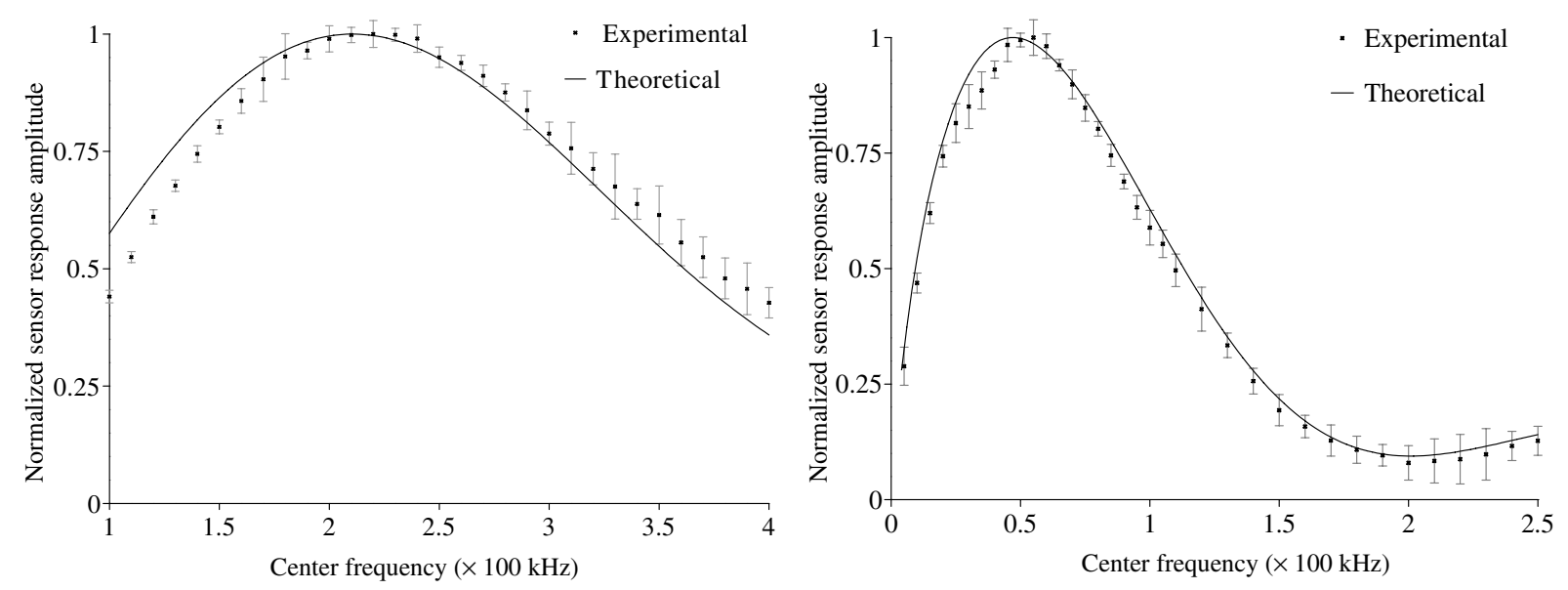

Figure 6. Comparison between experimental and theoretical sensor response amplitudes in the circular actuator experiment for (a) $\mathrm{S}_{0}$ mode (left) and (b) $\mathrm{A}_{0}$ mode (right).

the frequency range tested, i.e., the infinite plate assumption holds. Two sets of experiments were performed. In the first set, the actuators were excited in phase to excite symmetric GW modes, while in the second they were excited out of phase in order to excite the antisymmetric GW modes. These actuators were fed with a 3.5-cycle $9 \mathrm{~V}$ (peak-to-peak) Hannwindowed toneburst and the highest excitation frequency was well below the cut-off frequency of the first symmetric and first antisymmetric modes in each case. Thus, the $\mathrm{S}_{0} \mathrm{Lamb}$ mode was predominantly excited in the first set, while the $\mathrm{A}_{0}$ Lamb mode was predominantly excited in the second set. For each reading, the excitation signal was repeated at a frequency of $1 \mathrm{~Hz}$ (this was small enough so that there was no interference between successive repetitions) and the averaged signal over 64 samples was used to reduce the noise levels in the signal. Due to the piezo-actuator's capacitive behavior, its impedance varies with frequency, and so the actual voltage drop across it varies with frequency. To account for this, the voltage amplitude across the actuator terminals was also recorded for each reading and the sensor response amplitude and error estimate were compensated accordingly. To obtain the theoretical sensor response to a Hann-windowed toneburst at a given frequency, one needs to evaluate the inverse time domain Fourier transform over the excited frequency spectrum. The theoretical and experimental signal amplitudes, normalized by the peak amplitude over the tested frequency range, are compared over a range of frequencies for the $S_{0}$ and $\mathrm{A}_{0}$ modes in figure 6 . The error bars based on the standard deviation of the amplitudes over the 64 samples (capturing $99.73 \%$ of the data points) and normalized by the peak amplitude are also shown. The time-domain experimental and theoretical signals, also normalized to their respective peak amplitudes over the frequency range, are compared in figure 7 for certain center frequencies. A very similar experiment was done to explore the validity of the rectangular actuator derivation as shown in figure 5(b). In this case, an identical aluminum plate to the previous experiment was instrumented with $25 \mathrm{~mm} \times 5 \mathrm{~mm} \times 0.3 \mathrm{~mm}$ PZT-5A rectangular piezoactuators on either free surface at the center of the plate. Also, a $10 \mathrm{~mm} \times 10 \mathrm{~mm}$ PZT-5A piezo-sensor was mounted at a location $(3 \mathrm{~mm}, 35 \mathrm{~mm})$ relative to the plate center. Again, in this case, two sets of experiments for the $\mathrm{S}_{0}$ and $\mathrm{A}_{0} \mathrm{Lamb}$ modes were performed as described for the circular actuator experiment. The normalized theoretical and experimental amplitudes along with their associated error bars are compared for this experiment in figure 8 while the comparison of the normalized time domain signals is shown in figure 9 for certain center frequencies.

\section{Discussion and sources of error}

The normalized amplitude curves in figures 6 and 8 for both the circular and the rectangular actuator experiments match well with the theoretical predictions, both for predicting the peak frequency of response as well as the overall trend. There is a slight error observed in the prediction of the peak frequency in both cases, and this is more visible in the case of the rectangular actuator. This shift can be attributed to the shear lag phenomenon, which relates to the assumption made in the derivation pertaining to force transfer only along the free edges of the piezo. This 'pin-force' model was proposed by Crawley and de Luis [19] for the case of a pair of piezo-actuators surface bonded on opposite beam surfaces and actuated quasi-statically. In their formulation, the validity of the approximation depends on the shear lag parameter defined as

$$
\Gamma=\sqrt{\frac{a^{2} G_{b}\left(1+v_{a}\right)}{Y_{a}^{11} h_{a} h_{b}}\left(1+\frac{\eta Y_{a}^{11} h_{a}}{Y_{s} h_{s}}\right)}
$$

where $h_{b}$ is the bond layer thickness, $h_{a}$ is the actuator thickness, $h_{s}$ is the substrate thickness, $v_{a}$ is the actuator Poisson ratio, $G_{b}$ is the shear modulus of the bond layer, $Y_{s}$ is the substrate Young's modulus, $a$ is the actuator dimension and $\eta=2$ or 6 depending on whether the pair of actuators on either face of the beam is excited in the symmetric or antisymmetric actuation mode, respectively. It was proved that the 'pin-force' model assumption was perfect in the limit as $\Gamma$ approached infinity. For smaller $\Gamma$, due to the finite stiffness of the actuator relative to the plate and imperfect bonding between the actuator and plate, the force transfer between the piezo and the plate occurs over a finite length close to the edge of the piezo. Due to this, the effective 
Finite-dimensional piezoelectric transducer modeling for guided wave based structural health monitoring
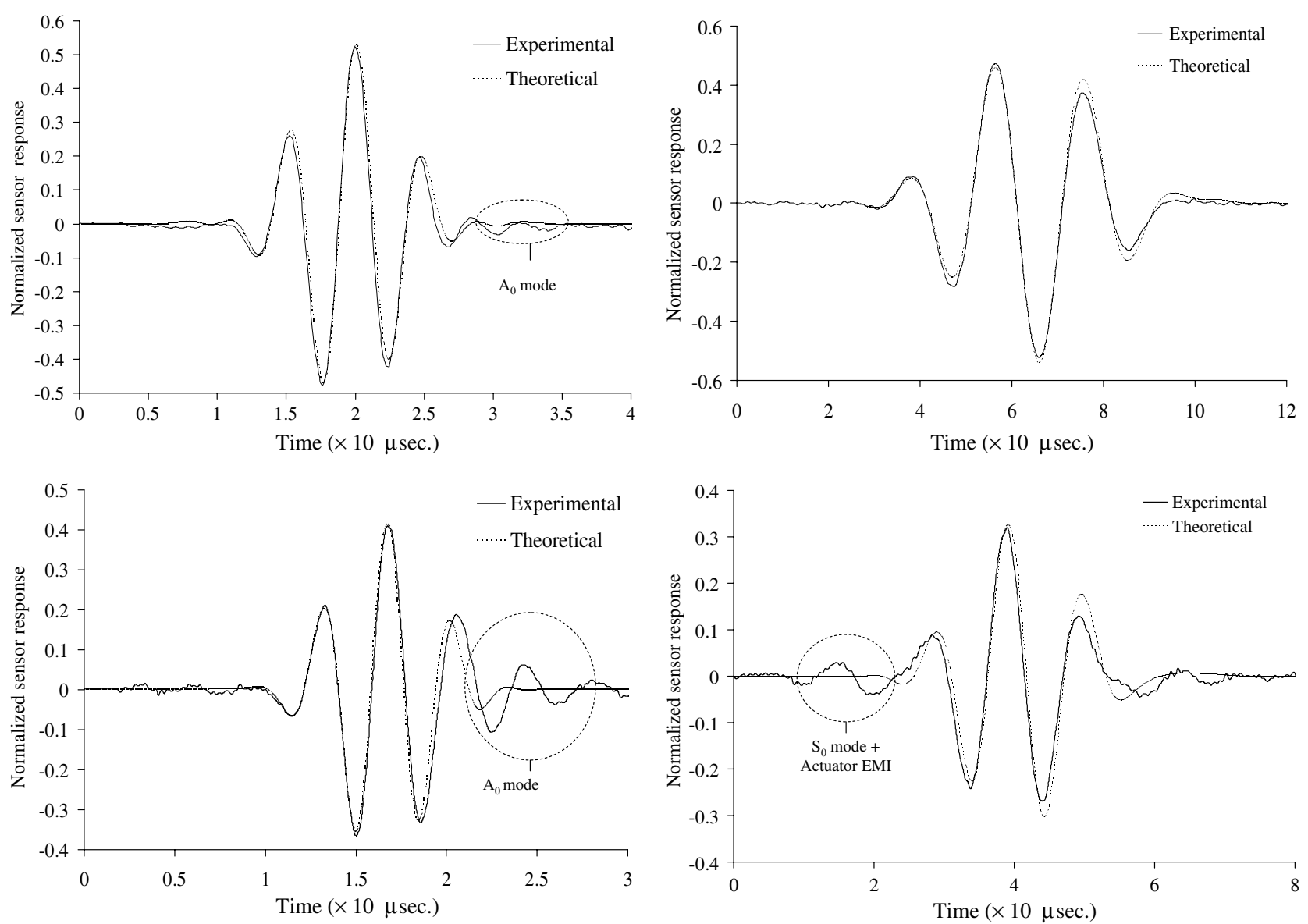

Figure 7. Comparison between experimental and theoretical sensor response time domain signals for the circular actuator experiment: (a) $\mathrm{S}_{0}$ mode (left) for the center frequencies (i) $200 \mathrm{kHz}$ and (ii) $300 \mathrm{kHz}$, and (b) $\mathrm{A}_{0}$ mode (right) for the center frequencies (i) $50 \mathrm{kHz}$ and (ii) $100 \mathrm{kHz}$.
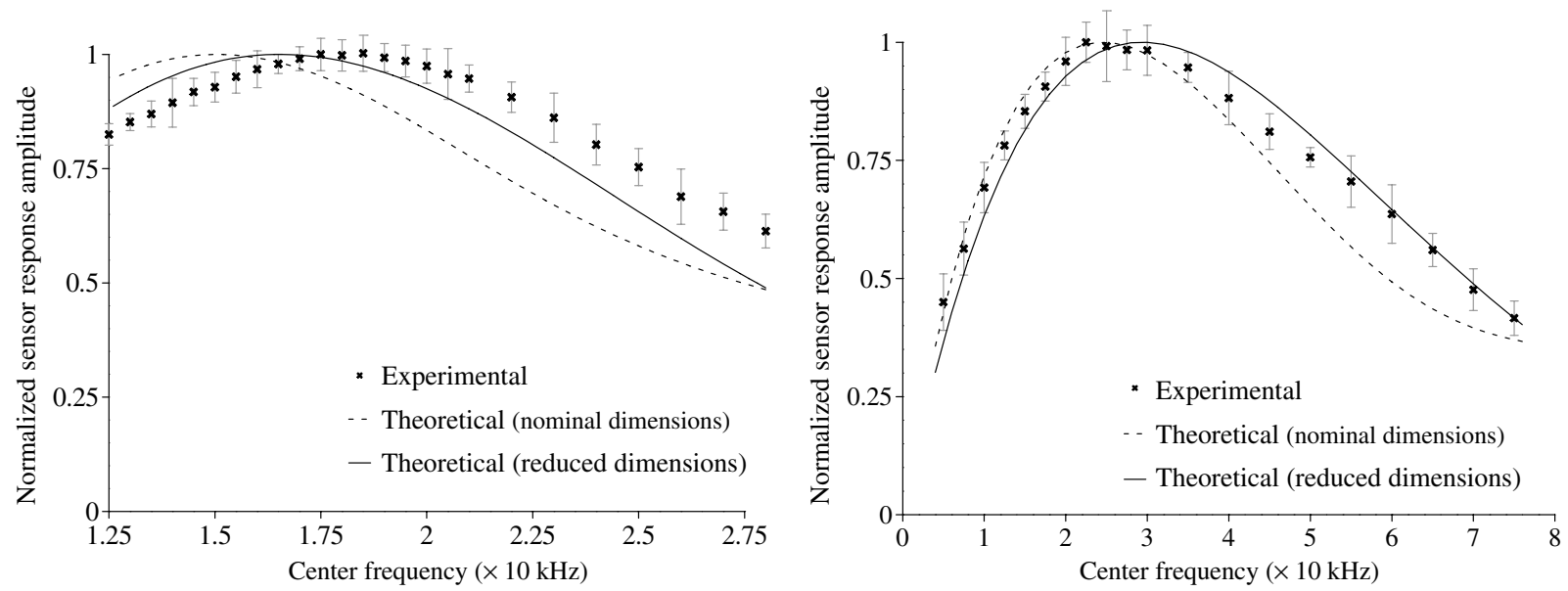

Figure 8. Comparison between experimental and theoretical sensor response amplitudes in the rectangular actuator experiment for (a) $\mathrm{S}_{0}$ mode (left) and (b) $A_{0}$ mode (right).

dimension of the piezo-actuator, $a$, in the models derived should be smaller than the actual physical dimension. While it was not possible to determine the shear modulus or thickness of the bond layer, assuming they were identical for both the rectangular and circular actuator experiments, it was calculated that the shear lag parameter for the circular actuator was 2.12 times greater than that for the rectangular actuator for both modes (assuming that the typical length for the rectangular actuator case is the dimension along the line joining the centers of the actuator and sensor), which explains the greater shift in 

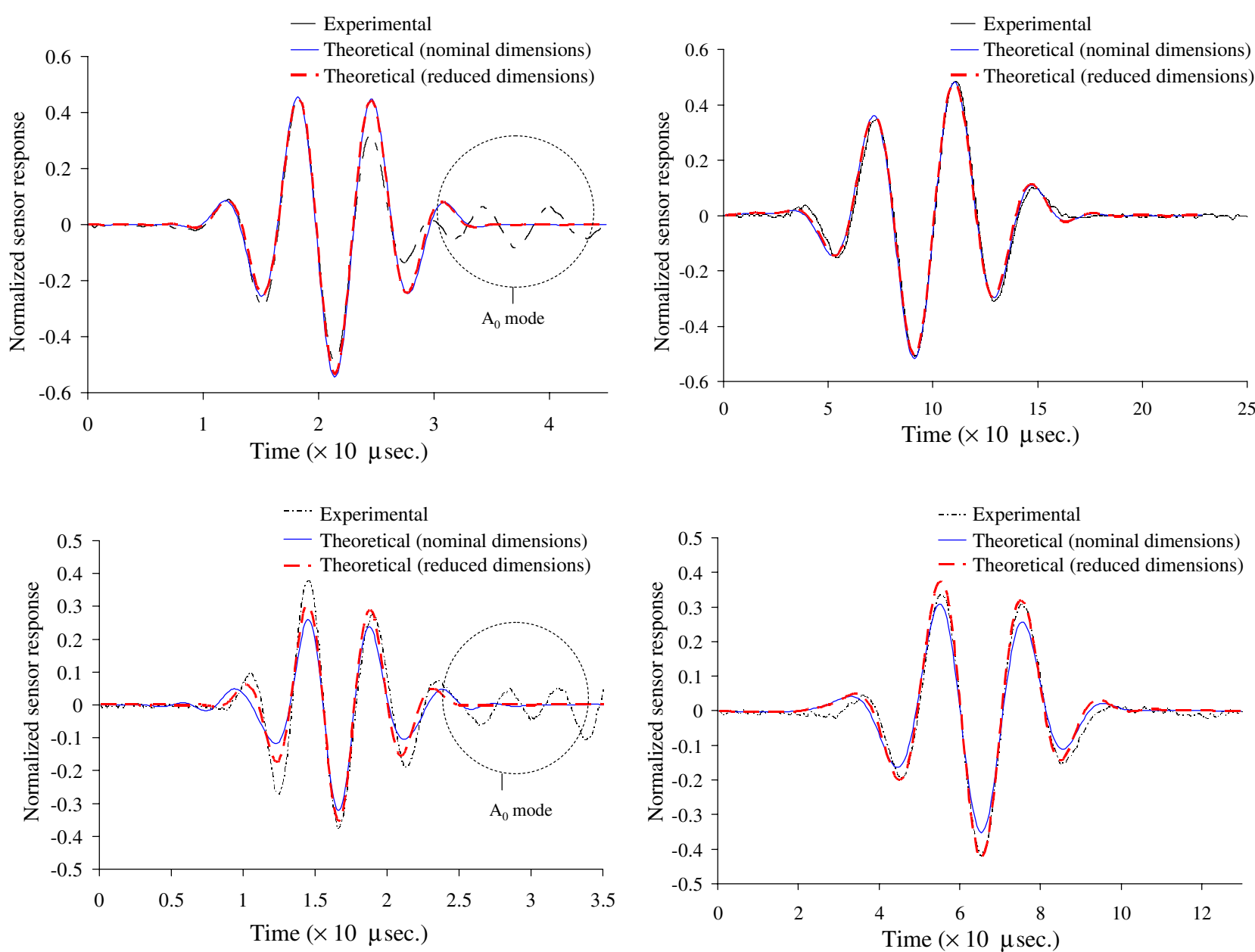

Figure 9. Comparison between experimental and theoretical sensor response time domain signals for the rectangular actuator experiment: (a) $\mathrm{S}_{0}$ mode (left) for the center frequencies (i) $150 \mathrm{kHz}$ and (ii) $250 \mathrm{kHz}$, and (b) $\mathrm{A}_{0}$ mode (right) for the center frequencies (i) $25 \mathrm{kHz}$ and (ii) $50 \mathrm{kHz}$.

the location of the peak frequency of response in the latter case. In the case of the rectangular actuator, the experimental curve is compared with two theoretical curves, one generated assuming the original physical actuator dimensions, and the other generated assuming $a_{1}=0.4 \mathrm{~cm}$ and $a_{2}=1 \mathrm{~cm}$, which is $20 \%$ smaller than the nominal physical dimensions. It is clear that the agreement with the experimental points is better in the case of the latter. The time domain plots also agree well with the experimental curves for their predicted modes in both cases. There was a small amplitude phantom signal observed in the sensor response during the actuation signal at the actuator due to some mild electro-magnetic interference (EMI). Also, while there were two actuators bonded on either free surface at the center of the plate in both cases, there is some mismatch in their piezoelectric properties due to manufacturing imperfections, as a result of which there was some excitation of antisymmetric modes in the symmetric mode experiments and vice versa. In addition, due to the finite thickness of the sensor, when the wavepacket is incident on it, due to scattering, a small portion of the incident $\mathrm{S}_{0}$ mode is converted to an $\mathrm{A}_{0}$ mode and vice versa. Hence, over certain frequency ranges, it was not possible to distinguish between the $\mathrm{A}_{0}$ and $\mathrm{S}_{0}$ mode signals, and these frequency ranges were avoided. As pointed out, in some of the $\mathrm{S}_{0}$ mode time domain signal plots the undesired $A_{0}$ mode signals can be seen following the $S_{0}$ mode signal predictions, and similarly for some of the $\mathrm{A}_{0}$ mode signals mild $S_{0}$ mode signals can be seen before the $A_{0}$ mode signal predictions.

Despite these sources of error, there is very good correlation between the experimental and theoretical results, thus providing validation for the derived models describing GW excitation due to surface-bonded circular and rectangular actuators on isotropic plates as well as the sensor response equation for surface-bonded piezo-sensors.

\section{Optimal sensor and actuator dimensions}

\subsection{The case of a circular actuator}

In this section, the optimal dimensions for the piezo-actuator and sensors to maximize sensor response are derived. Consider equation (43) for the harmonic sensor response of a piezo in the GW field due to a circular piezo-actuator. Assuming all 
Finite-dimensional piezoelectric transducer modeling for guided wave based structural health monitoring

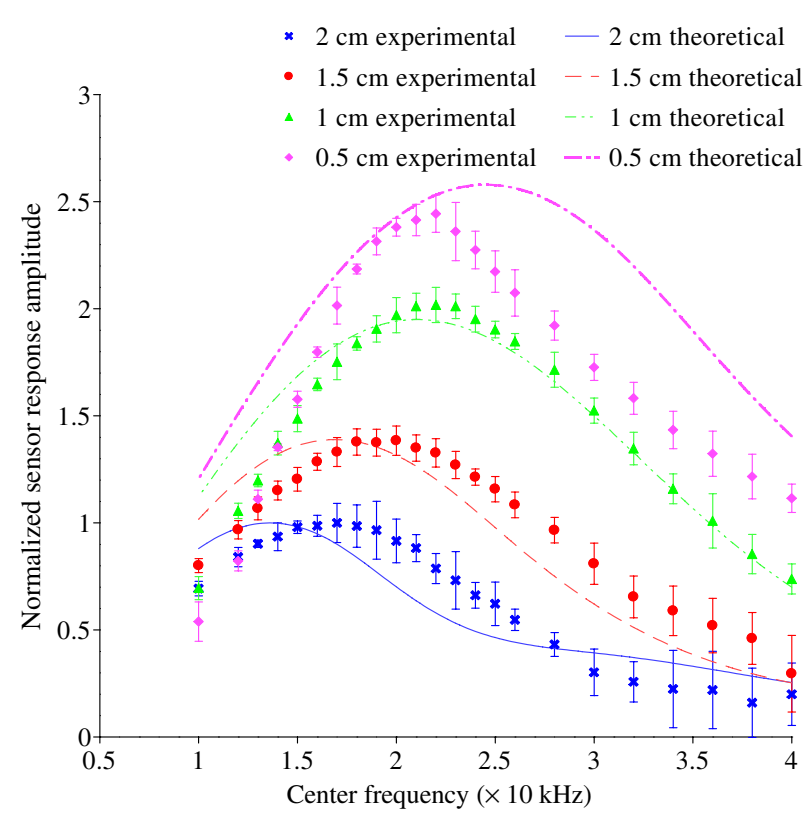

Figure 10. Comparison between experimental and theoretical sensor response amplitudes in the variable sensor length experiment.

parameters (except the sensor length $2 s_{r}$ ) to be constant,

$$
\left|V_{c}\right| \propto\left|\int_{r_{c}}^{r_{c}+2 s_{r}} \frac{H_{\mathrm{o}}^{(2)}(\xi r)}{2 s_{r}} \mathrm{~d} r\right| \leqslant \int_{r_{c}}^{r_{c}+2 s_{r}}\left|\frac{H_{0}^{(2)}(\xi r)}{2 s_{r}}\right| \mathrm{d} r .
$$

Since $\left|H_{0}^{(2)}(\xi r)\right|$ is a monotonically decreasing function of $r$,

$$
\left|H_{0}^{(2)}\left(\xi\left(r_{c}+2 s_{r}\right)\right)\right| \leqslant \int_{r_{c}}^{r_{c}+2 s_{r}}\left|\frac{H_{0}^{(2)}(\xi r)}{2 s_{r}}\right| \mathrm{d} r \leqslant\left|H_{0}^{(2)}\left(\xi r_{c}\right)\right|
$$

where the equality holds only at the limit of $2 s_{r} \rightarrow 0$. Thus, the maximum sensor response is attained for $2 s_{r}=0$, and it decreases with increasing $s_{r}$. This implies that the sensor should be as small as possible to maximize $\left|V_{c}\right|$ in the case of a circular-crested GW field. A smaller sensor size would also interfere less with the GW field and is favorable from the point of view of SHM system design, since the transducers should ideally occupy minimum structural area. To validate this idea, the same set-up as described in section 6 was used. However this time, a $20 \mathrm{~mm}$ (radial length) $\times 5 \mathrm{~mm}$ (width) $\times 0.3 \mathrm{~mm}$ (thickness) sensor was surface-bonded at a distance of radius $50 \mathrm{~mm}$ from the plate center so that $r_{c}=50 \mathrm{~mm}$, as before. The sensor's radial length $2 s_{r}$ was reduced in steps of $0.5 \mathrm{~cm}$ by cutting the sensor on the plate with a diamondpoint knife and examining the response of its remaining part. For each length, an experiment along the lines of the earlier ones was conducted for the $\mathrm{S}_{0}$ mode, i.e., the sensor response amplitudes were measured over 64 samples for each center frequency over a range of center frequencies. The comparison between the theoretical and experimental results is shown in figure 10 (both data sets are normalized to the peak value for the curve at $2 s_{r}=2 \mathrm{~cm}$ ). The theoretical curves were derived assuming uniform bond strength over all of the original piezosensor's area. As predicted by the theoretical model, the sensor response amplitude increases with decreasing sensor length.

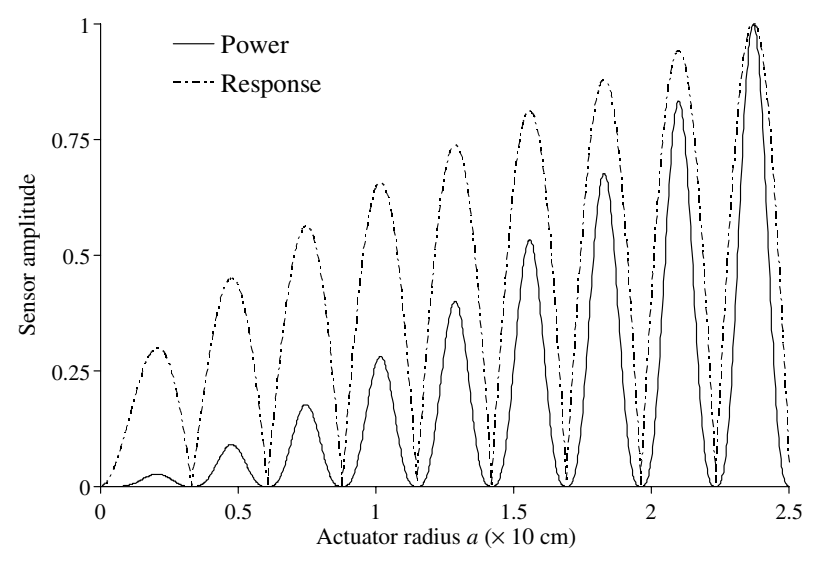

Figure 11. Amplitude variation of sensor response and power drawn to drive the acoustic field due to change in actuator radius for a $1 \mathrm{~mm}$ thick aluminum plate driven harmonically in the $S_{0}$ mode at $100 \mathrm{kHz}$.

The comparison between the theory and experimental results is good again, although the experimental curve is slightly shifted ahead of the theoretical curve along the center frequency axis due to shear lag. The comparison between the relative amplitudes is quite accurate with the exception of the last set for $2 s_{r}=0.5 \mathrm{~cm}$, which can be possibly attributed to weaker bond strength closer to the edge of the piezo-sensor.

To optimize the actuator size for maximum sensor response to harmonic excitation, as in the previous sub-section, everything in equation (43) is kept fixed except $a$. Then

$$
\left|V_{c}\right| \propto\left|a J_{1}(\xi a)\right| .
$$

The right-hand side of equation (49) is an oscillating function of $a$ with a monotonically increasing amplitude envelope as seen in figure 11. The local maxima of $\left|V_{c}\right|$ are attained at the corresponding local extrema of $J_{1}(\xi a)$. Thus, there is no optimum value for maximizing sensor response as such, and by choosing higher values of $a$ that yield local extrema one can in principle keep increasing the magnitude of sensor response to harmonic excitation. Notice that between any two successive peaks of the response function there is a value for the actuator radius for which the response to harmonic excitation is zero. This corresponds to a zero of the Bessel function. Although these zero 'nodes' caused by certain actuator radii are presented for simple harmonic excitation, they have also a direct impact on a toneburst signal. One may take a toneburst center frequency as responsible for most of the energy being delivered by the actuator. If the product of the actuator radius and the toneburst center frequency coincides with a node as shown in figure 11, then most of the signal will be attenuated.

Since the piezo-actuator has some capacitance, the harmonic reactive power circulated every cycle $P_{a}^{r}$ is

$$
P_{a}^{r}=2 \pi f C_{a} V_{a}^{2}=\frac{2 \pi f k_{a} \varepsilon_{0} \pi a^{2} V_{a}^{2}}{h_{a}} \propto a^{2}
$$

where $V_{a}$ is the actuation voltage supplied to the piezo-actuator, $f$ is the frequency of harmonic excitation and the rest of the notation is analogous to that used for the piezo-sensor. That is, the power circulation increases as the square of the 
actuator radius if all other parameters are constant. Note that the dependence of the capacitance on driving frequency and actuation voltage magnitude has not been considered (see [25], for example). However, these only tend to further increase the capacitance, and thereby the reactive power circulation. This power being reactive is not dissipated, but is merely used for charging the piezo in the positive half-cycle and is gained back when the capacitor is discharged in the negative half-cycle. The power supply to drive the actuators will define how much of this energy can be recycled.

In addition to this, the power source must supply the energy that is converted into acoustic energy in the excited GW field. This is given by the expression

$$
P_{a}^{d}=\int_{\mathrm{S}_{\mathrm{o}}} \hat{n} \cdot\left(u_{i} \sigma_{i j}\right) \mathrm{d} S
$$

where $\mathrm{S}_{\mathrm{o}}$ is the cylindrical surface of thickness $2 b$ and radius $a$ centered at the origin, i.e., that encapsulates the region of the plate under the actuator. On substituting the expressions for plate displacement and stress and evaluating the integral, an intricate expression is obtained involving the plate material properties, the plate thickness, the actuator radius, and the excitation frequency/wavenumber. Intuitively, however, one expects that this expression will also follow an oscillating trend with monotonically increasing amplitude envelope as a function of actuator radius. This is confirmed in figure 11, where the peaks of the expression coincide with the peaks of the sensor response curve. Evidently, the increased sensor response by actuator size tailoring is at the cost of increased power consumption by the actuator.

In summary, the choice of actuator length for the largest local maximum is limited by the power available to drive the actuator. Moreover, the area occupied by the actuator on the structure as well as the desired area covered by the actuatorsensor pair signal might be concerns that ultimately decide the actuator size.

\subsection{The case of a rectangular actuator}

Consider equation (45) for the harmonic sensor response of a piezo in the GW field due to a rectangular piezo-actuator. The far-field approximation for the harmonic sensor response using the method of stationary phase is

$$
\begin{aligned}
V_{c}^{S} & =\sum_{\xi^{S}} \frac{-8 \tau_{0} Y_{c}^{11} h_{c} g_{31}}{\pi \mu s_{1} s_{2}} \frac{N_{S}\left(\xi^{S}\right)}{\left(\xi^{S}\right)^{2} D_{S}^{\prime}\left(\xi^{S}\right)} \sqrt{\frac{2 \pi}{\xi^{S} r}}\left\{\left[\sin \left(\xi^{S} \cos \theta a_{1}\right)\right.\right. \\
& \left.\times \sin \left(\xi^{S} \sin \theta a_{2}\right) \sin \left(\xi^{S} \cos \theta c_{1}\right) \sin \left(\xi^{S} \sin \theta c_{2}\right)\right] \\
& \left.\times\left[\sin ^{2} 2 \theta\right]^{-1}\right\} \mathrm{e}^{-\mathrm{i}\left(\xi^{S} r+\pi / 4-\omega t\right)}
\end{aligned}
$$

where $\theta$ and $r$ are as defined in section 3. If all parameters except $s_{1}$ and $s_{2}$ are kept constant,

$$
\left|V_{c}^{S}\right| \propto\left|\frac{\sin \left(\xi^{S} \cos \gamma s_{1}\right)}{\left(\xi^{S} \cos \gamma s_{1}\right)} \frac{\sin \left(\xi^{S} \sin \gamma s_{2}\right)}{\left(\xi^{S} \sin \gamma s_{2}\right)}\right| .
$$

Since the function $\frac{\sin (t)}{(t)}$ is maximum at $t=0$, and its subsequent peaks after $t=0$ rapidly decay, one concludes that for maximum sensor response amplitude $\left(\left|V_{c}\right|\right)$ in the farfield the sensor dimensions, i.e., $2 s_{1}$ and $2 s_{2}$, should be as small as possible, preferably much smaller than the half-wavelength of the traveling wave.

For actuator size optimization, in the case of a rectangular actuator, due to the highly direction-dependent GW field, this will depend on the angular location of the piezo-sensor on the plate relative to the piezo-actuator. For example, consider the case $\theta=0$. Then the far-field sensor response can be shown to be (using L'Hospital's rule)

$$
\begin{aligned}
V_{c}^{S} & =\sum_{\xi^{S}} \frac{-16 \tau_{0} Y_{c}^{11} h_{c} g_{31}}{\pi \mu} \frac{N_{S}\left(\xi^{S}\right)}{D_{S}^{\prime}\left(\xi^{S}\right)} \\
& \times \sqrt{\frac{2 \pi}{\xi^{S} r}} \frac{a_{2} \sin \left(\xi^{S} a_{1}\right) \sin \left(\xi^{S} S_{1}\right)}{s_{1}} \mathrm{e}^{-\mathrm{i}\left(\xi^{S} r+\pi / 4-\omega t\right)} .
\end{aligned}
$$

If all parameters except $a_{1}$ and $a_{2}$ are kept constant, one obtains

$$
\left|V_{c}^{S}\right| \propto\left|a_{2} \sin \left(\xi^{S} a_{1}\right)\right| .
$$

Thus, to maximize the harmonic sensor response of a piezosensor in the direction $\theta=0, a_{2}$ should be as large as possible. For $a_{1}$, any of the lengths given by the relation

$$
2 a_{1}=\left(n+\frac{1}{2}\right) \frac{2 \pi}{\xi^{S}}, \quad n=0,1,2,3, \ldots
$$

are equally optimal values in order to maximize sensor response. By an analysis similar to the one in section 8.1, it can be shown that the power requirement increases for larger actuator dimensions. Thus, in order to minimize power consumption and the area occupied by the actuator on the structure, the value of $a_{1}$ is defined by equation (56) with $n=0$. The choice of $a_{2}$ will also be limited by similar concerns.

\section{Summary and conclusions}

In this work, a generic formulation to characterize the guided wave field excited by an arbitrary shape finite-dimensional surface-bonded piezoelectric wafer actuator was presented. It is based on the $3 \mathrm{D}$ linear elasticity equations for isotropic plates. This was then used to describe the generation of guided waves for the specific cases of ring-shaped and rectangular piezoelectric transducers. A model for circular actuators was developed as a special case of ring-shaped actuators, and this model was verified by FEM simulations. The piezoactuator was modeled as causing in-plane traction on the plate surface along its free edges, in the direction normal to the free edge. An expression for the output voltage response of a surface-bonded piezo-sensor in guided wave fields was also derived. Experiments seeking to investigate the accuracy of the models were conducted and the results of these were discussed for the fields excited by both circular and rectangular actuators. The experimental results were, in general, very consistent with the theoretical predictions based on the derived models, thus providing validation for the proposed models. The sources of error were examined in detail. Investigation of the actuator/sensor geometry for maximum performance was also done. It was found that, in both cases, the sensor size should be as small as possible to maximize the magnitude of its response to harmonic excitation, and this idea was proved experimentally for the circular actuator case. For circular actuators, it was found that there is no optimum 
actuator size although there are certain dimensions that lead to signal annihilation. For the case of rectangular actuators, the optimum actuator dimensions depend on the angular location of the piezo-sensor with respect to the piezo-actuator. Concerns such as area occupied by the actuator on the structure, desired area covered by the actuator-sensor pair signal, and power availability also need to be considered in deciding the actuator size in both cases.

\section{Acknowledgments}

The authors gratefully acknowledge suggestions from Dr Christopher Dunn (University of Michigan, presently Metis Design Corporation) for the experimental set-up. This work was supported by the Space Vehicle Technology Institute under grant NCC3-989 jointly funded by NASA and DoD within the NASA Constellation University Institutes Project, with Ms Claudia Meyer as the project manager.

\section{References}

[1] Guo N and Cawley P 1993 The interaction of Lamb waves with delaminations in composite laminates J. Acoust. Soc. Am. 94 2240-6

[2] Diaz Valdes S H and Soutis C 2000 Health monitoring of composites using Lamb waves generated by piezoelectric devices Plast. Rubber Compos. 29 475-81

[3] Culshaw B, Pierce S G and Staszekski W J 1998 Condition monitoring in composite materials: an integrated systems approach Proc. Inst. Mech. Eng. I 212 189-202

[4] Giurgiutiu V and Bao J 2002 Embedded ultrasonic structural radar with piezoelectric wafer active sensors for the NDE of thin-wall structures IMECE2002-33873: Proc. IMECE 2002: 2002 ASME Int. Mechanical Engineering Conr. (New Orleans, USA, Nov. 2002)

[5] Sundararaman S and Adams D E 2002 Phased transducer arrays for structural diagnostics through beamforming Proc. American Society for Composites 17th Technical Conf., Paper 177 (W. Lafayette, USA, Oct. 2002)

[6] Lemistre M and Balageas D 2001 Structural health monitoring system based on diffracted Lamb wave analysis by multiresolution processing Smart Mater. Struct. 10 504-11

[7] Rose J L 1999 Ultrasonic Waves in Solid Media (Cambridge: Cambridge University Press)
[8] Lamb H 1917 On waves in an elastic plate Proc. R. Soc. A 93 293-312

[9] Gazis D C 1958 Exact analysis of the plane-strain vibrations of thick-walled hollow cylinders J. Acoust. Soc. Am. 30 786-94

[10] Viktorov I A 1967 Rayleigh and Lamb Waves (New York: Plenum)

[11] Ditri J and Rose J L 1994 Excitation of guided waves in generally anisotropic layers using finite sources $J$. Appl. Mech. (Trans. ASME) $61330-8$

[12] Santosa F and Pao Y-H 1989 Transient axially asymmetric response of an elastic plate Wave Motion II 11 271-95

[13] Wilcox P 2004 Modeling the excitation of Lamb and SH waves by point and line sources Review of Quantitative Nondestructive Evaluation vol 23, ed D O Thompson and D E Chimenti pp 206-13

[14] Moulin E, Assaad J and Delebarre C 2000 Modeling of Lamb waves generated by integrated transducers in composite plates using a coupled finite element-normal modes expansion method J. Acoust. Soc. Am. 107 87-94

[15] Lin X and Yuan F G 2001 Diagnostic Lamb waves in an integrated piezoelectric sensor/actuator plate: analytical and experimental studies Smart Mater. Struct. 10 907-13

[16] Rose L R F and Wang C H 2004 Mindlin plate theory for damage detection: source solutions J. Acoust. Soc. Am. 116 154-71

[17] Veidt M, Liu T and Kitipornchai S 2001 Flexural waves transmitted by rectangular piezoceramic transducers Smart Mater. Struct. 10 681-8

[18] Giurgiutiu V 2003 Lamb wave generation with piezoelectric wafer active sensors for structural health monitoring Proc. SPIE 5056 111-22

[19] Crawley E F and de Luis J 1987 Use of piezoelectric actuators as elements of intelligent structures AIAA J. 25 1373-85

[20] González A and Alemany C 1996 Determination of the frequency dependence of characteristic constants in lossy piezoelectric materials J. Phys. D: Appl. Phys. 29 2476-82

[21] Miklowitz J 1978 The Theory of Elastic Waves and Waveguides (New York: North-Holland)

[22] Graff K F 1991 Wave Motion in Elastic Solids (New York: Dover)

[23] 2001 ABAQUS/Standard User's Manual Version 6.2 (Rhode Island, USA: Hibbitt, Karlsson and Sorensen, Inc.)

[24] 1988 IEEE Standard on Piezoelectricity ANSI/IEEE Std 176-1987 (New York: IEEE)

[25] Jordan T, Ounaies Z, Tripp J and Tcheng P 2000 Electrical properties and power considerations of a piezoelectric actuator NASA CR-2000-209861/ICASE Report No. 2000-8 